\title{
Regional Health Disparities in the Aftermath of Health System
}

\section{Reforms in Turkey}

\author{
Zeynep B. UĞUR ${ }^{1 *}$ \\ Abdullah TIRGíL ${ }^{2}$
}

Uğur, Z.B., Tirgil, A. (2021). Regional health disparities in the aftermath of health systems reforms in Turkey. Journal of Health Systems and Policies (JHESP), 3,137-169. DOI: 10.52675/jhesp.1004601

\section{ABSTRACT}

Starting in 2003, Turkey introduced major health system reforms through the Health Transformation Program (HTP). The HTP aimed to address inequities in health care services across Turkey. This study explores whether regional disparities in several health care indicators persist ten years after the HTP. For this purpose, we use administrative records and individuallevel data from the only regionally representative survey, Life Satisfaction Survey, undertaken by the Turkish Statistical Institute in 2013. First, the disadvantaged North-East, Middle-East, and South-East Anatolia regions experienced the most substantial increases in all health inputs and the odds of receiving the non-contributory Green Card insurance was also higher in these regions. Yet, there is still substantial disparity across regions in many health care access and satisfaction indicators. Especially, the disadvantaged regions still have lower utilization, lower satisfaction with healthcare, and lower subjective health scores in 2013 even after accounting for a wide range of control variables. While the HTP enabled the provision of a generous

\footnotetext{
${ }^{1}$ Social Sciences University of Ankara, Ankara, Turkey

${ }^{2}$ Ankara Yildirim Beyazit University, Ankara, Turkey

${ }^{*}$ Corresponding author: Z.B. UĞUR, zeynep.ugur@ asbu.edu.tr
} 
insurance benefits package, there is still room for progress in regional distribution of other major indicators such as subjective assessment of health, healthcare utilization, and satisfaction with health services. To further reduce health inequities across regions, the quality aspect of healthcare provision needs to be prioritized.

Keywords: Health Status Disparities, Access to Health Care, Health Care Utilization, Patient Satisfaction

\section{INTRODUCTION}

Worldwide, many countries are reforming their health systems to introduce or expand universal health coverage (UHC) to enhance access to health care for their citizens and to reduce financial hardship (Tangcharoensathien et al., 2011; Wagstaff et al., 2007). An important objective of these health system reforms is to improve equity in access and health outcomes by targeting poorer population groups (World Health Organization, 2010), often by strengthening primary health care services (Engström et al., 2001; Kutzin, 2013; Tirgil et al., 2018). Following the expansion of health insurance coverage in the USA, health services use has increased and catastrophic out-of-pocket health expenditures were reduced (Baicker et al., 2013). In Thailand, expansion of UHC through the 30 Baht Scheme helped to increase health care utilization, especially for the poorest population groups (Gruber et al., 2014). In Mexico, the introduction of Seguro Popular, the non-contributory health insurance for the poor helped to expand utilization of healthcare services and reduce catastrophic health expenditures (King et al., 2009; Knaul et al., 2018; Knaul et al., 2012).

From 2003 to 2012, Turkey introduced major health system reforms, the Health Transformation Program (HTP), to expand UHC and to promote equity and reduce disparities in access to healthcare services and health outcomes (Atun, 2015; Atun et al., 2013). These reforms, described elsewhere (Atun et al., 2013) and briefly summarized in Appendix A 
effectively targeted poor households by expanding the Green Card Scheme (a non-contributory insurance scheme for poor households) to more than 11 million people. Many studies have examined the effects of the expansion of UHC through HTP. Concerning efficiency, the reforms improved the productivity of the Ministry of Health hospitals (Sahin et al., 2011). As a result of the HTP, patient satisfaction with healthcare services in 2004-2012 is found to increase (Stokes et al., 2015). Patient satisfaction with healthcare services in the 2003-2017 by different insurance groups has also risen through the HTP related improvements (Ugur and Tirgil, 2018). In terms of equity, substantial improvements are recorded (Atun et al., 2013). Similarly, catastrophic health expenditures in the 2003-2009 period have been reduced with the HTP (Yardim et al., 2013). Also, it has been found that increasing benefits coverage for a noncontributory insurance scheme led to financial protection for low-income households by reducing out-of-pocket expenditures (Tirgil et al., 2019). On the other hand, the HTP had components such family practice services which operate with a profit margin that can contribute for the poor households to reduce their preventive care utilization. Earlier studies on the expansion of UHC in Turkey have analysed access to health care for the poor and the progressivity of out of pocket health expenditures to measure inequity. While improvement is demonstrated in access to healthcare services for women's health, children's vaccination and infant mortality across larger geographic areas (economically deprived east and richer west) (Atun et al., 2013), but whether UHC expansion helped to narrow regional disparities in the 81 provinces of Turkey have not been examined in detail.

The effect of HTP might be varied across regions that have different levels of socioeconomic development and there were historical inequities with regards to distribution of health resources (Hacettepe University Institute of Population Studies, 1994; Hacettepe University Institute of Population Studies, 2004). In Mexico, the health system reform had different effects depending on the demographic characteristics of insured households (Knaul et al., 2013) and 
again Mexico’s Seguro Popular was found to be more effective in urban areas (Grogger et al., 2014). A growing body of literature shows the effect of geographic proximity to healthcare facilities on utilization and health outcomes (Karra et al., 2016; Masters et al., 2013).

In this study, we examine utilization and satisfaction with health-care services in Turkey to evaluate the effect on different regions of health system reforms which led to expansion of UHC. We use administrative records and the Life Satisfaction Survey conducted in 2013 with approximately 196,000 observations. We examine how the users assess various dimensions of healthcare services, such as physician behavior, nurse behavior, organization and hygiene.

\section{METHODOLOGY}

Turkey has 81 provinces which are divided into 12 NUTS $^{3}-1$ regions. Our level of analysis in this study is these regions shown in Figure 1. For our analyses, administrative records of the number of physicians, hospital beds, and healthcare institutions belonging to the Ministry of Health are accessed from TurkStat and the Ministry of Health's Health Statistics Yearbooks. (Ministry of Health, 2010, 2014; TurkStat, 2013) The analysis is based on the 2003 and 2013 period as the HTP started as of 2003 and the main reforms were completed by 2012 .

Individual-level data from the Life Satisfaction Survey (LSS) conducted by the Turkish Statistical Institute (TurkStat) is utilized.(TurkStat, 2013a) The LSS is representative of Turkey's adult population (aged 18+ years). This annual survey has been implemented since 2003 through face to face interviews. In 2013, the survey was designed to have a representative sample from province and included a sample size of 196,203 individuals. The LSS 2013 is the only survey that allows studying regional disparities as it provides province information. The LSS covers a wide range of topics, including utilization of health-care services, insurance

\footnotetext{
${ }^{3}$ NUTS classification is a geocode standard for referencing the subdivisions of countries for statistical purposes established by Eurostat.
} 
coverage, satisfaction with health-care services, and a large number of background indicators on the socio-economic characteristics of individuals participating in the survey. We focus on three distinct outcome measures, including subjective health assessment, health-care utilization, and satisfaction with health-care services. People are asked to assess their health status from 1 (fully satisfied) to 5 (not satisfied at all). Our measure of health-care utilization comes from a question in which respondents were asked whether or not they have used health-care services in the last 12 months. We restrict our measure of satisfaction with health-care services to only those who used health-care services in the last 12 months in order to minimize recall bias, measured using the answers to the following question: "Are you satisfied with health care services?" ranging from 1 "fully satisfied" to 5 "not satisfied at all." The survey also provides information on insurance status, including whether an individual was part of the Green Card Scheme for the poor. Our measure of access is the degree of insurance coverage.

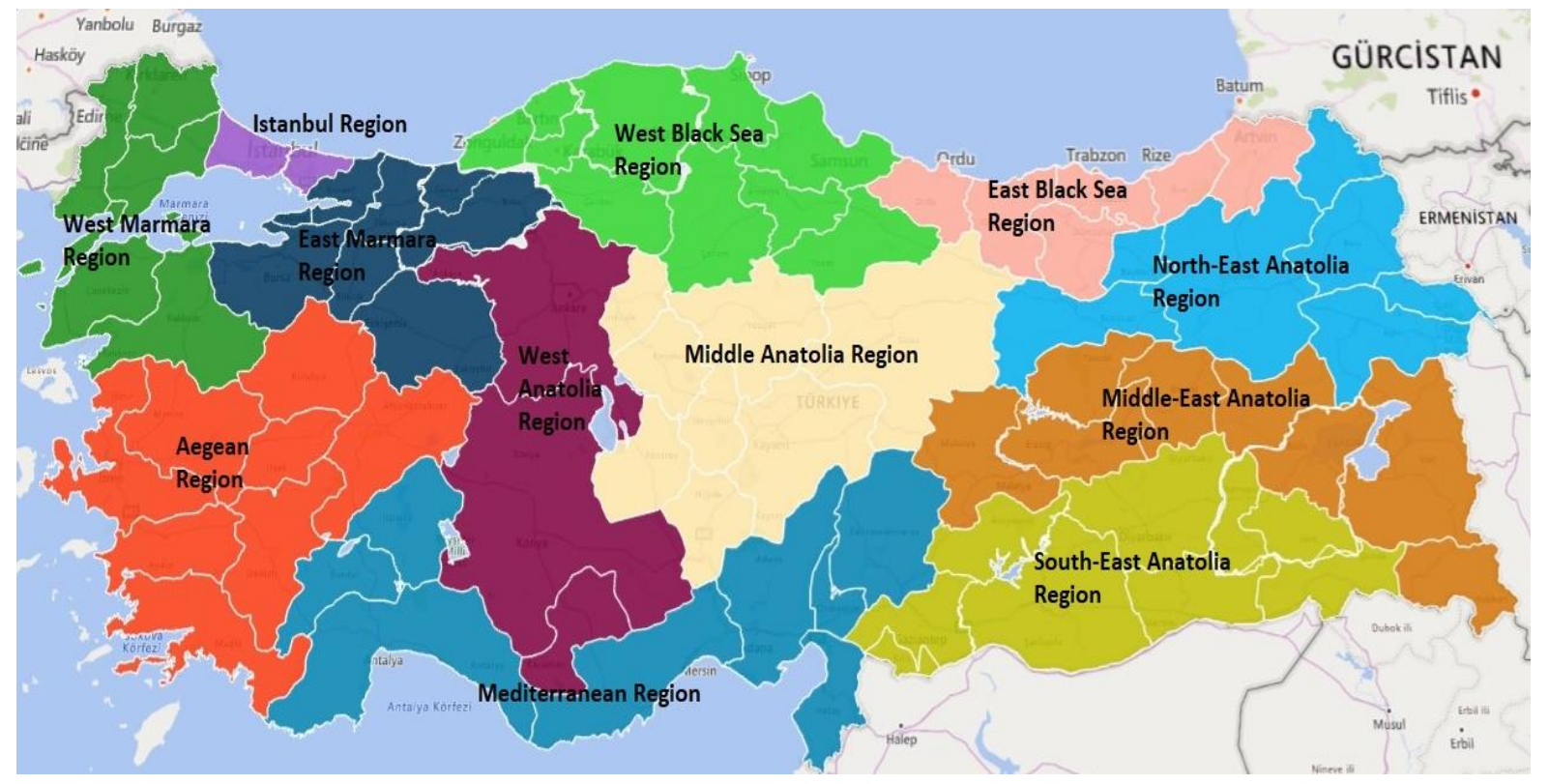

Figure 1: Turkey’s Regions at NUTS 1 level

The LSS also asked many questions that allow controlling for a broad range of background variables. The respondents' gender, age, education level, marital status and employment status are available. Moreover, respondents' satisfaction with their friends is also added to account for the general disposition of the individual. Besides, from the household 
module, we can obtain the household income in 5 brackets, in which the individual income is not available.

To detect the problems in health services in different dimensions, several questions were administered, such as issues with physician's behaviour, nurse's behaviour, hygiene, trouble getting an appointment, and perceiving co-payments as non-affordable. Respondents can answer these questions as "Yes," "No," or "Do not know". We removed those who replied as "Do not know" for our control variables (age, gender, education level, marital status, and employment status).

To study the regional disparities in various health indicators, the following equations are used:

$y_{i}=\beta_{0}+A X_{i}+B R_{r}+\varepsilon_{i}$,

$y_{i}=\beta_{0}+A X_{i}+B R_{r}+C I_{i}+\varepsilon_{i}$

$y_{i}=\beta_{0}+\boldsymbol{A} X_{i}+B R_{r}+C I_{i}+D S_{r}+\varepsilon_{i}$,

where $y_{i}$ can be a health indicator such as subjective health assessment, health care utilization in the last 12 months, and satisfaction with health care services. $X_{i}$ denotes background characteristics for individuals; $R_{r}$ is regional fixed effects, consisting of 12 regions; $I_{i}$ is insurance type fixed effects, composed of government employee retirement fund, social security institution, insurance for self-artisans, private insurance, green card; $S_{r}$ indicates regional supply-side variables, which are the number of physicians per thousand persons, the number of public facilities per thousand persons, and the number of beds in public facilities per thousand persons. The main variables of interest are region dummy variables.

STATA 12.0 software is used for data analysis and P-value $<0.05$ is used as a cut-off for significance analyses. As this study uses secondary data, it is not necessary to obtain ethics approval and thus, we did not obtain ethics approval. 


\section{RESULTS}

Figure 2 shows the percentage change in health-care inputs in 2003-2013. Appendix Table B-1 provides the details of the change in health inputs across regions over time (See Appendix Figure 10 to 13 for more details). Figure 2 shows the most substantial increase $(110 \%)$ in the South-East Anatolia region in terms of the number of physicians. The MiddleEast Anatolia region also achieves a 70\% increase in the number of physicians. For the NorthEast Anatolia region, we observe a considerable increase in all three health inputs. East and West Marmara regions also benefit from increased inputs as the number of physicians are raised by $64 \%$ and $65 \%$, number of beds are raised by $32 \%$ and $4 \%$, respectively.

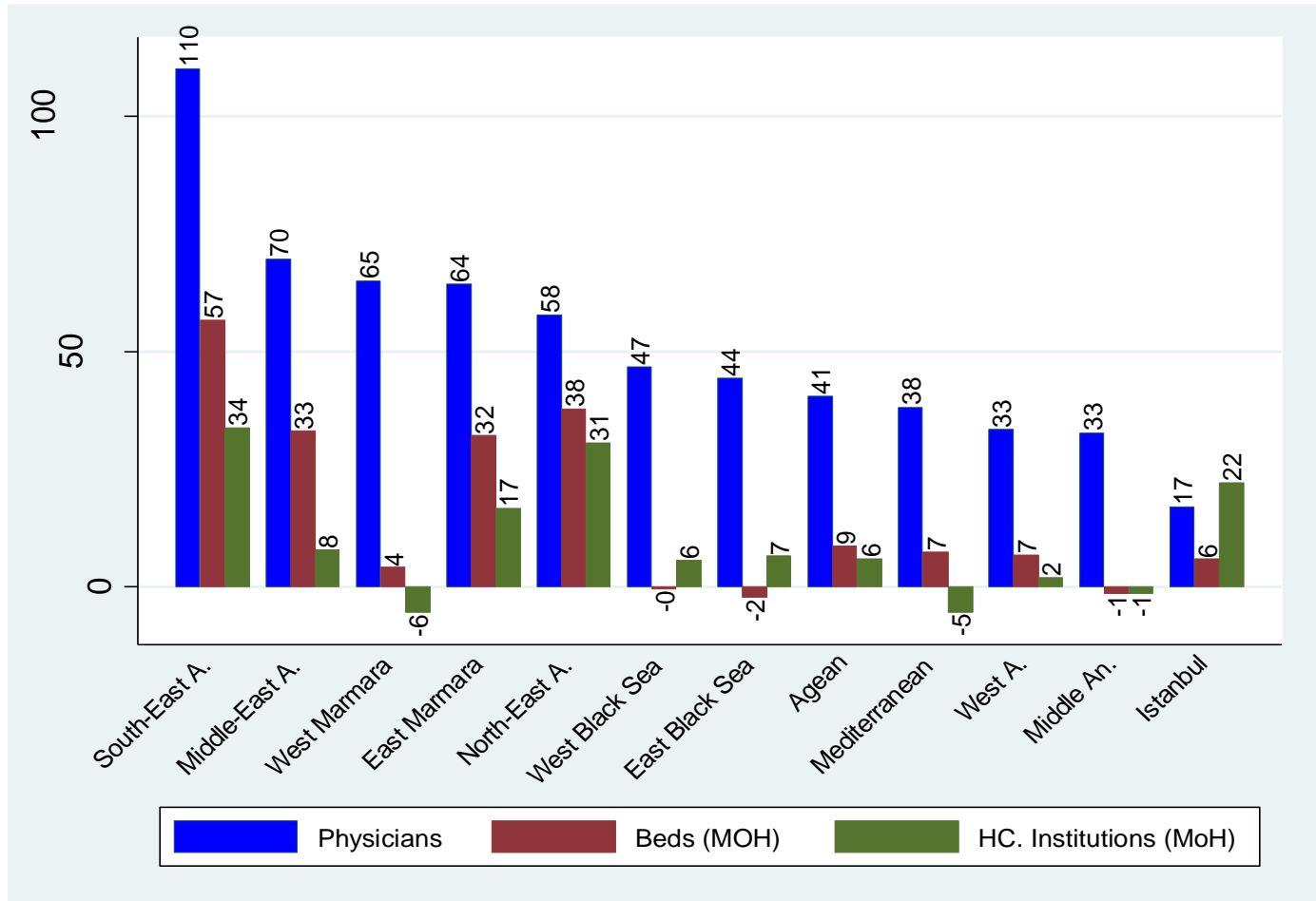

Figure 2: \% Change in Healthcare Resources between 2003-2013 from TurkStat (2013b)

Istanbul region benefits the least in terms of the number of physicians, whereas Middle Anatolia experiences a $1 \%$ decline for both the number of health-care institutions and the number of hospital beds. In Mediterranean region, the number of health-care institutions declines by $5 \%$ and in East-Black Sea region, the number of hospital beds shrinks by $2 \%$. 
Table 1 provides summary statistics for the sample. The first row shows mean values and standard deviations for the whole sample, and the following 12 rows present the results for the 12 regions of Turkey. We conduct a one-way ANOVA to determine if health-care indicators were different for regions. Overall satisfaction with one's health for the whole sample out of 5 is 3.57, which implies that people are somewhat satisfied with their health. There is a statistically significant difference across regions in terms of average satisfaction with health as determined by the one-way ANOVA. When we analyze the percentage of people who are either not satisfied at all with their health or not satisfied, we also observe a statistically significant difference across regions according to the one-way ANOVA test results. We observe that $24 \%$ $(\mathrm{SD}=0.43)$ of people living in South-East Anatolia, 21\% $(\mathrm{SD}=0.41)$ of those living in NorthEast Anatolia, and 22\% ( $\mathrm{SD}=0.41)$ of those living in Middle-East Anatolia regions have the highest proportion of respondents in terms of unsatisfactory health assessment. The one-way ANOVA test result reveals a significant and well-defined difference across regions with respect to the utilization of health care services. The three regions with the highest level of poor health (South-East Anatolia, North-East Anatolia, and Middle-East Anatolia) are also the ones with the lowest level of health-care service utilization with 62\% ( $\mathrm{SD}=0.49), 62 \%(\mathrm{SD}=0.49)$, and $61 \%(\mathrm{SD}=0.49)$, respectively. Similarly, the test results indicates statistically significant differences in terms of satisfaction with health-care services across regions. South-East Anatolia, the North-East Anatolia, and the Middle-East Anatolia regions have considerably lower satisfaction with health-care services. Another notable difference across regions is the access to health-care services measured by those not having insurance coverage and those having a green card. South-East Anatolia, North-East Anatolia, and Middle-East Anatolia regions also have higher rates of uninsured individuals whereas, South-East Anatolia ( $\mathrm{M}=33 \%$, $\mathrm{SD}=0.47)$, North-East Anatolia $(\mathrm{M}=28 \%, \mathrm{SD}=0.45)$ and Middle-East Anatolia $(\mathrm{M}=36 \%$, $\mathrm{SD}=0.48$ ) tended to have higher portions of Green Card holders. 
Table 1: Health-care Services Indicators across Regions Notes: mean coefficients; sd in brackets, ${ }^{*} p<0.05,{ }^{* *} p<0.01,{ }^{* * *} p<0.001$. The analysis is for the 12 regions of Turkey.

\begin{tabular}{|c|c|c|c|c|c|c|c|}
\hline & $\begin{array}{l}\text { Satisfaction w. } \\
\text { Health }\end{array}$ & $\begin{array}{c}\text { Unsatisfied w. } \\
\text { Health }\end{array}$ & $\begin{array}{c}\text { Utilization of } \\
\text { HCS }\end{array}$ & $\begin{array}{c}\text { Satisfaction w. } \\
\text { HCS }\end{array}$ & $\begin{array}{l}\text { No Health } \\
\text { Insurance }\end{array}$ & Green Card & $N$ \\
\hline Total & $\begin{array}{c}3.57 \\
{[0.89]} \\
\end{array}$ & $\begin{array}{c}0.16 \\
{[0.37]} \\
\end{array}$ & $\begin{array}{c}0.69 \\
{[0.46]} \\
\end{array}$ & $\begin{array}{c}3.66 \\
{[0.85]} \\
\end{array}$ & $\begin{array}{c}0.08 \\
{[0.27]}\end{array}$ & $\begin{array}{c}0.12 \\
{[0.32]}\end{array}$ & 196,203 \\
\hline South-East An. & $\begin{array}{c}3.41 \\
{[0.99]}\end{array}$ & $\begin{array}{c}0.24 \\
{[0.43]}\end{array}$ & $\begin{array}{c}0.62 \\
{[0.49]}\end{array}$ & $\begin{array}{c}3.60 \\
{[0.87]}\end{array}$ & $\begin{array}{c}0.11 \\
{[0.31]}\end{array}$ & $\begin{array}{c}0.33 \\
{[0.47]}\end{array}$ & 19,225 \\
\hline North-East A. & $\begin{array}{c}3.50 \\
{[1.03]}\end{array}$ & $\begin{array}{c}0.21 \\
{[0.41]}\end{array}$ & $\begin{array}{c}0.62 \\
{[0.49]}\end{array}$ & $\begin{array}{c}3.63 \\
{[0.96]}\end{array}$ & $\begin{array}{c}0.12 \\
{[0.32]}\end{array}$ & $\begin{array}{c}0.28 \\
{[0.45]}\end{array}$ & 10,077 \\
\hline Middle-East A. & $\begin{array}{c}3.49 \\
{[1.00]}\end{array}$ & $\begin{array}{c}0.22 \\
{[0.41]}\end{array}$ & $\begin{array}{c}0.61 \\
{[0.49]}\end{array}$ & $\begin{array}{c}3.50 \\
{[1.03]}\end{array}$ & $\begin{array}{c}0.08 \\
{[0.27]}\end{array}$ & $\begin{array}{c}0.36 \\
{[0.48]}\end{array}$ & 15,552 \\
\hline West Marmara & $\begin{array}{c}3.66 \\
{[0.81]}\end{array}$ & $\begin{array}{c}0.12 \\
{[0.32]}\end{array}$ & $\begin{array}{c}0.69 \\
{[0.46]}\end{array}$ & $\begin{array}{c}3.71 \\
{[0.78]}\end{array}$ & $\begin{array}{c}0.07 \\
{[0.25]}\end{array}$ & $\begin{array}{c}0.05 \\
{[0.22]}\end{array}$ & 13,885 \\
\hline Aegean & $\begin{array}{c}3.64 \\
{[0.83]}\end{array}$ & $\begin{array}{c}0.13 \\
{[0.34]}\end{array}$ & $\begin{array}{c}0.71 \\
{[0.46]}\end{array}$ & $\begin{array}{c}3.73 \\
{[0.79]}\end{array}$ & $\begin{array}{c}0.08 \\
{[0.28]}\end{array}$ & $\begin{array}{c}0.04 \\
{[0.20]}\end{array}$ & 26,465 \\
\hline East Marmara & $\begin{array}{c}3.64 \\
{[0.82]}\end{array}$ & $\begin{array}{c}0.13 \\
{[0.34]}\end{array}$ & $\begin{array}{c}0.68 \\
{[0.47]}\end{array}$ & $\begin{array}{c}3.67 \\
{[0.82]}\end{array}$ & $\begin{array}{c}0.06 \\
{[0.24]}\end{array}$ & $\begin{array}{c}0.02 \\
{[0.13]}\end{array}$ & 19,690 \\
\hline West An. & $\begin{array}{c}3.63 \\
{[0.82]}\end{array}$ & $\begin{array}{c}0.14 \\
{[0.34]}\end{array}$ & $\begin{array}{c}0.76 \\
{[0.43]}\end{array}$ & $\begin{array}{c}3.62 \\
{[0.82]}\end{array}$ & $\begin{array}{c}0.08 \\
{[0.26]}\end{array}$ & $\begin{array}{c}0.04 \\
{[0.20]}\end{array}$ & 12,862 \\
\hline Mediterranean & $\begin{array}{c}3.51 \\
{[0.88]}\end{array}$ & $\begin{array}{c}0.17 \\
{[0.38]}\end{array}$ & $\begin{array}{c}0.72 \\
{[0.45]}\end{array}$ & $\begin{array}{c}3.63 \\
{[0.82]}\end{array}$ & $\begin{array}{c}0.08 \\
{[0.27]}\end{array}$ & $\begin{array}{c}0.10 \\
{[0.30]}\end{array}$ & 25,101 \\
\hline Middle An. & $\begin{array}{c}3.57 \\
{[0.92]}\end{array}$ & $\begin{array}{c}0.17 \\
{[0.37]}\end{array}$ & $\begin{array}{c}0.72 \\
{[0.45]}\end{array}$ & $\begin{array}{c}3.74 \\
{[0.79]}\end{array}$ & $\begin{array}{c}0.07 \\
{[0.26]}\end{array}$ & $\begin{array}{c}0.08 \\
{[0.27]}\end{array}$ & 16,278 \\
\hline West Black S. & $\begin{array}{c}3.60 \\
{[0.91]}\end{array}$ & $\begin{array}{c}0.16 \\
{[0.36]}\end{array}$ & $\begin{array}{c}0.72 \\
{[0.45]}\end{array}$ & $\begin{array}{c}3.78 \\
{[0.81]}\end{array}$ & $\begin{array}{c}0.06 \\
{[0.23]}\end{array}$ & $\begin{array}{c}0.06 \\
{[0.24]}\end{array}$ & 17,515 \\
\hline East Black S. & $\begin{array}{c}3.57 \\
{[0.82]}\end{array}$ & $\begin{array}{c}0.15 \\
{[0.36]}\end{array}$ & $\begin{array}{c}0.72 \\
{[0.45]}\end{array}$ & $\begin{array}{c}3.79 \\
{[0.67]}\end{array}$ & $\begin{array}{c}0.06 \\
{[0.23]}\end{array}$ & $\begin{array}{c}0.09 \\
{[0.28]}\end{array}$ & 7,344 \\
\hline Istanbul & $\begin{array}{c}3.68 \\
{[0.85]}\end{array}$ & $\begin{array}{c}0.12 \\
{[0.33]}\end{array}$ & $\begin{array}{c}0.70 \\
{[0.46]}\end{array}$ & $\begin{array}{c}3.53 \\
{[0.95]}\end{array}$ & $\begin{array}{c}0.08 \\
{[0.27]}\end{array}$ & $\begin{array}{c}0.02 \\
{[0.12]}\end{array}$ & 12,209 \\
\hline F Test & 144.30 & 188.84 & 161.63 & 178.29 & 69.44 & 2706.81 & \\
\hline P value & 0.00 & 0.00 & 0.00 & 0.00 & 0.00 & 0.00 & \\
\hline
\end{tabular}


Table 2 presents regression results for subjective health. The first three columns of Table 2 show the results for one's subjective health (one's health satisfaction) which are estimated using OLS models for the sake of ease of interpretation of coefficients. We also provide ordered probit estimates in Appendix B Table 2 for the same models which are very close to OLS estimates. The 4th-6th columns show the logit regression results (odds ratios) for being dissatisfied with one's health dummy variable.

According to Model 1 and Model 4 in Table 2, there are significant differences with regard to subjective health across regions after controlling for a large set of background variables. Results suggest that people living in North-East, Middle-East, and South-East Anatolia have lower subjective health scores and are more likely to be dissatisfied with their health compared to people living in West Marmara, East Marmara, Aegean, West Anatolia, West Black Sea, and East Black Sea. In Model 2 and Model 5, we included four dummy variables for each of the insurance schemes (Social Insurance Organization for formal sector employees, Government Employees Retirement Fund for retired civil servants, Active Civil Servants Insurance Fund for civil servants in work and their dependents, Bağ-Kur for artisans, self-employed and agricultural workers and Green Card Scheme for the poor) with the reference category of no health insurance to account for the differential health insurance holding across regions. In Model 3 and 6, healthcare inputs are added as additional control variables to examine whether differences in subjective health indicators stem from differences in the numbers of health-care facilities and personnel across regions.

What we observe from these results is that adding additional controls produce lower coefficient estimates for the subjective health for the regions South-East Anatolia, Middle-East Anatolia, North-East Anatolia, and the Mediterranean and higher coefficients for many other 
regions, which indicates differences in insurance and supply-side variables across regions. For the rest of the regions, it appears that controlling health insurance categories and supply-side interventions indicate higher satisfaction. Another significant result is that satisfaction with health for those in Istanbul showed no statistically significant point estimates for Model 1 and Model 2, where only baseline characteristics and health insurance categories are included in the regression analyses. However, when supply-side information is added to the regressions, we observe a 7-percentage point higher subjective health assessment for people living in Istanbul compared to people living in Middle Anatolia. Nevertheless, there are still persistent differences in subjective health across regions.

Table 3 presents regression results for the utilization of healthcare services in the last 12 months (the first three columns) and satisfaction with health care services (the last three columns). The first three columns provide odds ratios for utilized healthcare dummy variables, and the last three columns provide OLS estimates of satisfaction with healthcare services on a 5-point scale. (Appendix B Table B-2 provides ordered probit estimates which essentially leads to similar conclusions) What stands out from the utilization results is that North-East, MiddleEast, and South-East regions utilization is statistically significantly lower than Middle Anatolia even in Model 3, which controls for differences in health insurance and healthcare inputs. When we examine other regions, we see a concentration of the odds of 1 with some insignificant differences (Aegean, Istanbul, West Black Sea, and East Black Sea regions), significantly lower (East and West Marmara regions) and significantly higher (West Anatolia, Mediterranean regions) utilizations. These differences suggest that there might be cultural differences across regions that prevent people from North-East, Middle-East, and South-East regions. 
Table 2: Regression Results for Subjective Health

\begin{tabular}{|c|c|c|c|c|c|c|}
\hline & $\begin{array}{c}\text { SWH } \\
\text { (1) }\end{array}$ & $\begin{array}{c}\text { SWH } \\
\text { (2) }\end{array}$ & $\begin{array}{c}\text { SWH } \\
(3)\end{array}$ & $\begin{array}{l}\text { DDV-OR } \\
\text { (4) }\end{array}$ & $\begin{array}{c}\text { DDV-OR } \\
\text { (5) }\end{array}$ & $\begin{array}{c}\text { DDV-OR } \\
\text { (6) }\end{array}$ \\
\hline $\begin{array}{l}\text { South-East An. } \\
\text { (ref: Middle An.) }\end{array}$ & $\begin{array}{c}-0.17^{\text {**** }} \\
(0.01)\end{array}$ & $\begin{array}{c}- \\
0.14^{* * *} \\
(0.01)\end{array}$ & $\begin{array}{c}-0.12^{* * *} \\
(0.01)\end{array}$ & $\begin{array}{l}1.70^{* * *} \\
(0.05)\end{array}$ & $\begin{array}{l}1.55^{* * * *} \\
(0.05)\end{array}$ & $\begin{array}{l}1.45^{* * * *} \\
(0.05)\end{array}$ \\
\hline Middle-East An. & $\begin{array}{c}-0.13^{* * *} \\
(0.01)\end{array}$ & $\begin{array}{c}- \\
0.10^{* * *} \\
(0.01)\end{array}$ & $\begin{array}{c}-0.11^{\text {*** }} \\
(0.01)\end{array}$ & $\begin{array}{l}1.62^{* * * *} \\
(0.05)\end{array}$ & $\begin{array}{l}1.45^{\text {**** }} \\
(0.05)\end{array}$ & $\begin{array}{l}1.50^{* * *} \\
(0.05)\end{array}$ \\
\hline North-East An. & $\begin{array}{c}-0.08^{* * *} \\
(0.01)\end{array}$ & $\begin{array}{c}- \\
0.05^{* * *} \\
(0.01)\end{array}$ & $\begin{array}{c}-0.06^{* * *} \\
(0.01)\end{array}$ & $\begin{array}{l}1.42^{* * * *} \\
(0.05)\end{array}$ & $\begin{array}{l}1.30^{* * * *} \\
(0.05)\end{array}$ & $\begin{array}{l}1.31^{* * * *} \\
(0.05)\end{array}$ \\
\hline Mediterranean & $\begin{array}{c}-0.04^{* * * *} \\
(0.01)\end{array}$ & $\begin{array}{c}- \\
0.04^{* * * *} \\
(0.01)\end{array}$ & $\begin{array}{l}-0.01 \\
(0.01)\end{array}$ & $\begin{array}{c}1.02 \\
(0.03)\end{array}$ & $\begin{array}{c}1.01 \\
(0.03)\end{array}$ & $\begin{array}{l}0.92^{\text {** }} \\
(0.03)\end{array}$ \\
\hline West Marmara & $\begin{array}{l}0.08^{* * *} \\
(0.01)\end{array}$ & $\begin{array}{l}0.08^{* * *} \\
(0.01)\end{array}$ & $\begin{array}{l}0.10^{* * *} \\
(0.01)\end{array}$ & $\begin{array}{l}0.65^{* * *} \\
(0.02)\end{array}$ & $\begin{array}{l}0.66^{* * *} \\
(0.02)\end{array}$ & $\begin{array}{l}0.63^{* * *} \\
(0.02)\end{array}$ \\
\hline East Marmara & $\begin{array}{l}0.03^{* * *} \\
(0.01)\end{array}$ & $\begin{array}{l}0.03^{* * *} \\
(0.01)\end{array}$ & $\begin{array}{l}0.04^{* * *} \\
(0.01)\end{array}$ & $\begin{array}{l}0.85^{* * *} \\
(0.03)\end{array}$ & $\begin{array}{l}0.85^{* * *} \\
(0.03)\end{array}$ & $\begin{array}{l}0.82^{* * *} \\
(0.03)\end{array}$ \\
\hline Istanbul & $\begin{array}{l}-0.00 \\
(0.01)\end{array}$ & $\begin{array}{c}0.00 \\
(0.01)\end{array}$ & $\begin{array}{l}0.07^{* * * *} \\
(0.01)\end{array}$ & $\begin{array}{c}0.96 \\
(0.04)\end{array}$ & $\begin{array}{c}0.95 \\
(0.04)\end{array}$ & $\begin{array}{l}0.78^{* * *} \\
(0.04)\end{array}$ \\
\hline Aegean & $\begin{array}{l}0.08^{* * *} \\
(0.01)\end{array}$ & $\begin{array}{l}0.08^{* * * *} \\
(0.01)\end{array}$ & $\begin{array}{l}0.12^{* * * *} \\
(0.01)\end{array}$ & $\begin{array}{l}0.73^{* * *} \\
(0.02)\end{array}$ & $\begin{array}{l}0.74^{* * * *} \\
(0.02)\end{array}$ & $\begin{array}{l}0.67^{* * *} \\
(0.02)\end{array}$ \\
\hline West An. & $\begin{array}{l}0.02^{* *} \\
(0.01)\end{array}$ & $\begin{array}{l}0.02^{* *} \\
(0.01)\end{array}$ & $\begin{array}{l}0.10^{* * *} \\
(0.01)\end{array}$ & $\begin{array}{l}0.90^{* * *} \\
(0.03)\end{array}$ & $\begin{array}{l}0.90^{* * *} \\
(0.03)\end{array}$ & $\begin{array}{l}0.74^{* * *} \\
(0.03)\end{array}$ \\
\hline West Black Sea & $\begin{array}{l}0.05^{* * *} \\
(0.01)\end{array}$ & $\begin{array}{l}0.05^{* * *} \\
(0.01)\end{array}$ & $\begin{array}{l}0.04^{* * * *} \\
(0.01)\end{array}$ & $\begin{array}{l}0.87^{* * *} \\
(0.03)\end{array}$ & $\begin{array}{l}0.87^{* * *} \\
(0.03)\end{array}$ & $\begin{array}{l}0.89^{* * * *} \\
(0.03)\end{array}$ \\
\hline East Black Sea & $\begin{array}{l}0.06^{* * * *} \\
(0.01)\end{array}$ & $\begin{array}{l}0.07^{* * * *} \\
(0.01)\end{array}$ & $\begin{array}{l}0.06^{* * *} \\
(0.01)\end{array}$ & $\begin{array}{l}0.75^{* * *} \\
(0.03)\end{array}$ & $\begin{array}{l}0.75^{* * *} \\
(0.03)\end{array}$ & $\begin{array}{l}0.78^{* * * *} \\
(0.03)\end{array}$ \\
\hline Health Insurance & - & + & + & - & + & + \\
\hline Healthcare Inputs & - & - & + & - & - & + \\
\hline Baseline controls & + & + & + & + & + & + \\
\hline $\begin{array}{l}\text { R-squared } \\
\mathrm{N}\end{array}$ & $\begin{array}{c}0.142 \\
196,203\end{array}$ & $\begin{array}{l}0.146 \\
192,875\end{array}$ & $\begin{array}{l}0.146 \\
192,875\end{array}$ & $\begin{array}{l}0.111 \\
196,203\end{array}$ & $\begin{array}{l}0.114 \\
192,875\end{array}$ & $\begin{array}{l}0.115 \\
192,875\end{array}$ \\
\hline
\end{tabular}

Notes: SWH stands for Satisfaction with Health, DDV stands for Dissatisfied Dummy Variable. Std. errors are clustered at household level and provided in ()$, * * * \mathrm{p}<0.01, * * \mathrm{p}<0.05, * \mathrm{p}<0.10$. The reference category is Middle Anatolia region which includes the Aksaray, Kayresi, Kırıkkale, Kırşehir, Nevşehir, Niğde, Sivas and Yozgat provinces. All models include baseline controls. Baseline Controls: gender, age, age squared, 5 household income bracket dummy variables, employment status dummy variables (categories: employed, unemployed, doing house-care, student, retired, unable to work, other employment status), marital status dummy variables (categories: married, divorced/separated, widowed, single), educational attainment dummy variables (categories: primary school or less, secondary school graduate, high school graduate, university or more), and satisfaction from relationship with friends. Health insurance categories include Social Insurance Organization for formal sector employees, Government Employees Retirement Fund for retired civil servants, Active Civil Servants Insurance Fund for civil servants in work and their dependents, Bağ-Kur for artisans, self-employed and agricultural workers and Green Card Scheme for the poor with the reference category of no insurance. Healthcare inputs include number of physicians per thousand persons, number of public healthcare institutions per thousand persons, number of public hospital beds per thousand persons. 


\begin{tabular}{lcccccc}
\hline & HCU-OR & HCU-OR & HCU-OR & SHS & SHS & SHS \\
& $(\mathbf{1})$ & $(\mathbf{2})$ & $(\mathbf{3})$ & $\mathbf{( 4 )}$ & $\mathbf{( 5 )}$ & $(\mathbf{6})$ \\
\hline South-East An. & $0.67^{* * *}$ & $0.70^{* * *}$ & $0.72^{* * *}$ & $-0.12^{* * *}$ & $-0.11^{* * *}$ & $-0.07^{* * *}$ \\
(ref: Middle An.) & $(0.02)$ & $(0.02)$ & $(0.02)$ & $(0.01)$ & $(0.01)$ & $(0.01)$ \\
Middle-East An. & $0.66^{* * *}$ & $0.68^{* * *}$ & $0.68^{* * *}$ & $-0.25^{* * *}$ & $-0.24^{* * *}$ & $-0.27^{* * *}$ \\
& $(0.02)$ & $(0.02)$ & $(0.02)$ & $(0.01)$ & $(0.01)$ & $(0.01)$ \\
North-East An. & $0.66^{* * *}$ & $0.70^{* * *}$ & $0.69^{* * * *}$ & $-0.13^{* * *}$ & $-0.12^{* * *}$ & $-0.13^{* * *}$ \\
& $(0.02)$ & $(0.02)$ & $(0.02)$ & $(0.01)$ & $(0.01)$ & $(0.01)$ \\
Mediterranean & $1.05^{*}$ & $1.05^{* *}$ & 1.04 & $-0.08^{* * *}$ & $-0.08^{* * *}$ & $-0.03^{* * * *}$ \\
& $(0.03)$ & $(0.03)$ & $(0.03)$ & $(0.01)$ & $(0.01)$ & $(0.01)$ \\
West Marmara & $0.93^{* * *}$ & $0.93^{* *}$ & $0.89^{* * * *}$ & $-0.05^{* * *}$ & $-0.05^{* * *}$ & $-0.04^{* * *}$ \\
& $(0.03)$ & $(0.03)$ & $(0.03)$ & $(0.01)$ & $(0.01)$ & $(0.01)$ \\
East Marmara & $0.81^{* * *}$ & $0.81^{* * *}$ & $0.80^{* * *}$ & $-0.06^{* * *}$ & $-0.06^{* * *}$ & $-0.05^{* * *}$ \\
& $(0.02)$ & $(0.02)$ & $(0.02)$ & $(0.01)$ & $(0.01)$ & $(0.01)$ \\
Istanbul & $0.92^{* * *}$ & $0.94^{* *}$ & $0.94^{*}$ & $-0.17^{* * *}$ & $-0.17^{* * *}$ & $-0.05^{* * *}$ \\
& $(0.03)$ & $(0.03)$ & $(0.03)$ & $(0.01)$ & $(0.01)$ & $(0.01)$ \\
Aegean & 0.97 & 0.98 & $0.95^{*}$ & $-0.03^{* * *}$ & $-0.02^{* * *}$ & $0.02^{* * *}$ \\
& $(0.02)$ & $(0.02)$ & $(0.03)$ & $(0.01)$ & $(0.01)$ & $(0.01)$ \\
West An. & $1.19^{* * *}$ & $1.20^{* * *}$ & $1.13^{* * *}$ & $-0.08^{* * *}$ & $-0.08^{* * *}$ & 0.02 \\
& $(0.04)$ & $(0.04)$ & $(0.04)$ & $(0.01)$ & $(0.01)$ & $(0.01)$ \\
West Black Sea & 1.02 & 1.00 & 0.98 & -0.00 & -0.00 & $-0.03^{* * *}$ \\
& $(0.03)$ & $(0.03)$ & $(0.03)$ & $(0.01)$ & $(0.01)$ & $(0.01)$ \\
East Black Sea & 1.00 & 0.97 & $0.94^{*}$ & $0.03^{* * *}$ & $0.03^{* *}$ & -0.01 \\
& $(0.03)$ & $(0.03)$ & $(0.03)$ & $(0.01)$ & $(0.01)$ & $(0.01)$ \\
\hline Health Insurance & - & + & + & - & + & + \\
\hline Healthcare Inputs & - & - & + & - & - & + \\
\hline Baseline controls & + & + & + & + & + & + \\
\hline R-squared & 0.062 & 0.071 & 0.071 & 0.111 & 0.114 & 0.115 \\
N & 196,203 & 192,875 & 192,875 & 196,203 & 192,875 & 192,875 \\
\hline
\end{tabular}

Table 3: Regression Results for Utilization and Satisfaction

Notes: HCU stands for Health Care Utilization, SHS stands for Satisfaction with Health Services. Std. errors are clustered at household level and provided in ( ), *** $\mathrm{p}<0.01, * * \mathrm{p}<0.05, * \mathrm{p}<0.10$. The reference category is Middle Anatolia region which includes the Aksaray, Kayresi, Kırıkkale, Kırşehir, Nevşehir, Niğde, Sivas and Yozgat provinces. All models include baseline controls and 5 point scaled subjective health assessment. Health insurance categories include Social Insurance Organization for formal sector employees, Government Employees Retirement Fund for retired civil servants, Active Civil Servants Insurance Fund for civil servants in work and their dependents, Bağ-Kur for artisans, self-employed and agricultural workers and Green Card Scheme for the poor with the reference category of no insurance. Healthcare inputs include number of physicians per thousand persons, number of public healthcare institutions per thousand persons, number of public hospital beds per thousand persons. 


\begin{tabular}{|c|c|c|c|c|}
\hline & $\begin{array}{l}\text { GC-OR } \\
\text { (1) }\end{array}$ & $\begin{array}{l}\text { GC-OR } \\
\text { (2) }\end{array}$ & $\begin{array}{c}\text { NOINS-OR } \\
\text { (3) }\end{array}$ & $\begin{array}{c}\text { NOINS-OR } \\
\text { (4) }\end{array}$ \\
\hline $\begin{array}{l}\text { South-East An. } \\
\text { (ref: Middle An.) }\end{array}$ & $\begin{array}{l}3.74^{* * *} \\
(0.17)\end{array}$ & $\begin{array}{l}3.41^{* * *} \\
(0.16)\end{array}$ & $\begin{array}{c}1.02 \\
(0.05)\end{array}$ & $\begin{array}{c}1.00 \\
(0.05)\end{array}$ \\
\hline Middle-East An. & $\begin{array}{l}5.32^{* * * *} \\
(0.26)\end{array}$ & $\begin{array}{l}5.15^{* * *} \\
(0.25)\end{array}$ & $\begin{array}{l}0.76^{* * *} \\
(0.04)\end{array}$ & $\begin{array}{l}0.76^{* * *} \\
(0.04)\end{array}$ \\
\hline North-East An. & $\begin{array}{l}3.86^{* * *} \\
(0.20)\end{array}$ & $\begin{array}{l}4.04^{* * * *} \\
(0.21)\end{array}$ & $\begin{array}{l}1.35^{* * *} \\
(0.08)\end{array}$ & $\begin{array}{l}1.36^{* * *} \\
(0.08)\end{array}$ \\
\hline Mediterranean & $\begin{array}{l}1.25^{* * *} \\
(0.06)\end{array}$ & $\begin{array}{l}1.14^{* * * *} \\
(0.06)\end{array}$ & $\begin{array}{l}1.08 \\
(0.05)\end{array}$ & $\begin{array}{l}1.06 \\
(0.05)\end{array}$ \\
\hline West Marmara & $\begin{array}{l}0.70^{* * * *} \\
(0.05)\end{array}$ & $\begin{array}{c}0.68^{* * * *} \\
(0.05)\end{array}$ & $\begin{array}{c}1.04 \\
(0.06)\end{array}$ & $\begin{array}{c}1.03 \\
(0.06)\end{array}$ \\
\hline East Marmara & $\begin{array}{l}0.27^{* * *} \\
(0.02)\end{array}$ & $\begin{array}{l}0.28^{* * * *} \\
(0.02)\end{array}$ & $\begin{array}{c}1.02 \\
(0.06)\end{array}$ & $\begin{array}{l}1.03 \\
(0.06)\end{array}$ \\
\hline Istanbul & $\begin{array}{l}0.29^{* * * *} \\
(0.03)\end{array}$ & $\begin{array}{l}0.26^{* * *} \\
(0.03)\end{array}$ & $\begin{array}{l}1.45^{* * *} \\
(0.08)\end{array}$ & $\begin{array}{l}1.41^{* * * *} \\
(0.08)\end{array}$ \\
\hline Aegean & $\begin{array}{l}0.55^{* * *} \\
(0.03)\end{array}$ & $\begin{array}{l}0.51^{* * *} \\
(0.03)\end{array}$ & $\begin{array}{l}1.38^{* * * *} \\
(0.07)\end{array}$ & $\begin{array}{l}1.35^{* * *} \\
(0.07)\end{array}$ \\
\hline West An. & $\begin{array}{l}0.70^{* * *} \\
(0.05)\end{array}$ & $\begin{array}{l}0.65^{* * *} \\
(0.05)\end{array}$ & $\begin{array}{l}1.32^{* * * *} \\
(0.08)\end{array}$ & $\begin{array}{l}1.30^{* * *} \\
(0.08)\end{array}$ \\
\hline West Black Sea & $\begin{array}{l}0.85^{* * *} \\
(0.05)\end{array}$ & $\begin{array}{l}0.82^{* * * *} \\
(0.05)\end{array}$ & $\begin{array}{l}0.88^{* *} \\
(0.05)\end{array}$ & $\begin{array}{l}0.88^{* *} \\
(0.05)\end{array}$ \\
\hline East Black Sea & $\begin{array}{c}1.09 \\
(0.07)\end{array}$ & $\begin{array}{c}1.07 \\
(0.07)\end{array}$ & $\begin{array}{l}0.77^{* * * *} \\
(0.05)\end{array}$ & $\begin{array}{l}0.77^{* * * *} \\
(0.05)\end{array}$ \\
\hline GDP per capita & & $\begin{array}{l}0.07^{* * * *} \\
(0.02)\end{array}$ & & $\begin{array}{l}0.55^{*} \\
(0.17)\end{array}$ \\
\hline Baseline controls & + & + & + & + \\
\hline $\begin{array}{l}\text { R-squared } \\
\mathrm{N}\end{array}$ & $\begin{array}{c}0.324 \\
192,875\end{array}$ & $\begin{array}{l}0.325 \\
192,875\end{array}$ & $\begin{array}{l}0.105 \\
192,875\end{array}$ & $\begin{array}{l}0.105 \\
192,875\end{array}$ \\
\hline
\end{tabular}

Table 4: Regression Results for Access to Healthcare

Notes: GC stands for Green Card Ownership, NOINS stands for having No Health Insurance dummy variable. Std. errors are clustered at household level and provided in ( ), *** $\mathrm{p}<0.01, * * \mathrm{p}<0.05, * \mathrm{p}<0.10$. The reference category is Middle Anatolia region which includes the Aksaray, Kayresi, Kırıkkale, Kırşehir, Nevşehir, Niğde, Sivas, and Yozgat provinces. All models include baseline controls, and 5 points scaled subjective health assessment.

As shown in the last three columns of Table 3, there are also significant variations across regions about satisfaction with healthcare services. Again, these three regions (North-East, Middle-East, and South-East) have lower satisfaction with health services. Istanbul is observed to have the second-largest negative coefficient in terms of satisfaction with health services. 
When we account for health insurance and health inputs, we observe a considerable reduction in the coefficient of the South-East region in terms of dissatisfaction with health care services. However, we do not observe a drop in the coefficients of North-East and Middle-East regions.

Table 4 presents regression results for access to health care measured by the noncontributory green card ownership dummy variable (the first two columns) and having no health insurance dummy variable (the last two columns). The first two columns provide odds ratios for Green Card ownership, and the columns 3-4 provide odd ratios for having no health insurance. The first two columns present strong evidence that people in North-East, MiddleEast, and South-East are more likely to have a green card after accounting for their income and many other background characteristics. We observe that those people living in the generally affluent regions of Turkey, such as Istanbul, Aegean, Marmara are less likely to hold a Green Card. The results in the last two columns also show that people in South-East are not statistically different from people in Middle Anatolia in terms of having no insurance, and people in the Middle-East Anatolia region are less likely to have no health insurance. However, we still observe a higher likelihood of having no insurance in the North-East Anatolia region. Columns 3-4 of Table 4 show that many region coefficients are not statistically significant. Also, Istanbul, Aegean and West Anatolia present a higher likelihood of having no health insurance.

Although North-East, South-East, and Middle-East regions are the regions that benefited the most from the HTP, we do not observe higher utilization nor satisfaction with healthcare services in these regions. To examine the differences in the quality of health care inputs, we study people's self-reported problems in various domains of health care services across regions. We also provide the distribution of problems across provinces in Appendix Figure B-1 to B-8. 
In Figure 3, we report the percentage of people experiencing problems related to an appointment across regions. We observe higher discrepancy rates across regions about appointment waiting times and less discrepancy concerning appointment taking. $49 \%$ of people in the Middle-East, $41 \%$ of people in South-East and $40 \%$ of people in North-East Anatolia have problems in appointment waiting times. Interestingly, Istanbul is the region with $39 \%$ of people reporting appointment waiting problems, and that has the highest percentage of people who experience problems in appointment taking (27\% of people). Except for Istanbul, MiddleEast, South-East, and North-East Anatolia are again the highest regions for experiencing problems in terms of getting an appointment.

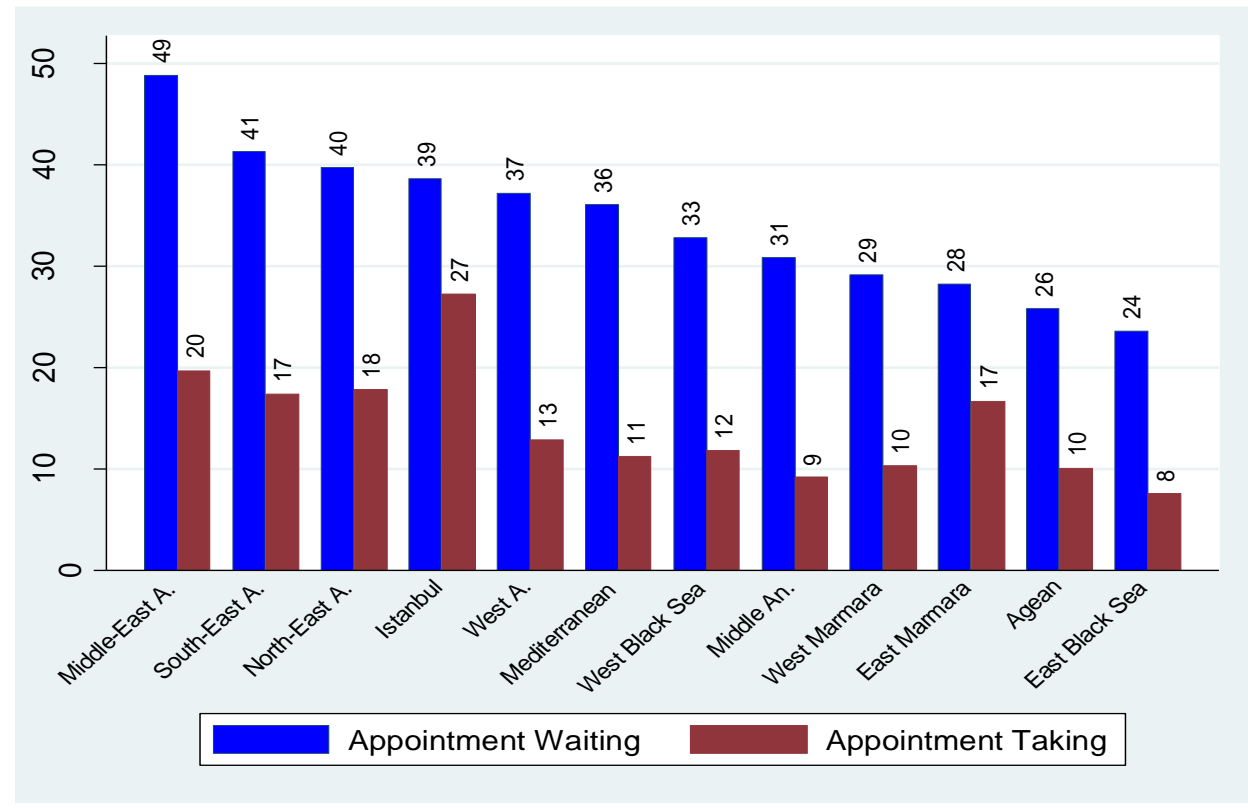

Figure 3: Problems related to Appointment by Region

Figure 4 presents the breakdown of problems with shortages of health care staff and lack of hygiene. The range of people who finds health care staff insufficient varies between 61 to $43 \%$, with an average of $52 \%$. That implies that the inadequacy of health care personnel is a prevalent problem for all regions. Hygiene issues are lesser of a problem as people having issues 
with hygiene is approximately $15 \%$ ranging from $11 \%$ to $24 \%$. As in the same line with Figure 3, Middle-East, South-East, North-East Anatolia, and Istanbul are the highest regions for considering inadequate health care staff and experiencing problems in terms of hygiene.

Another aspect of measuring the quality of health care services is the behaviour of health care personnel to their patients. Figure 5 provides the percentages of people who find physician's and nurse's behaviour problematic. We observe fewer people experiencing trouble in terms of health care staffs' behaviour, and the discrepancy between regions is mild. However, three regions (North-East Anatolia, Istanbul, and Middle-East) except South-East Anatolia are again the highest regions for having problems due to health care staffs' behaviour.

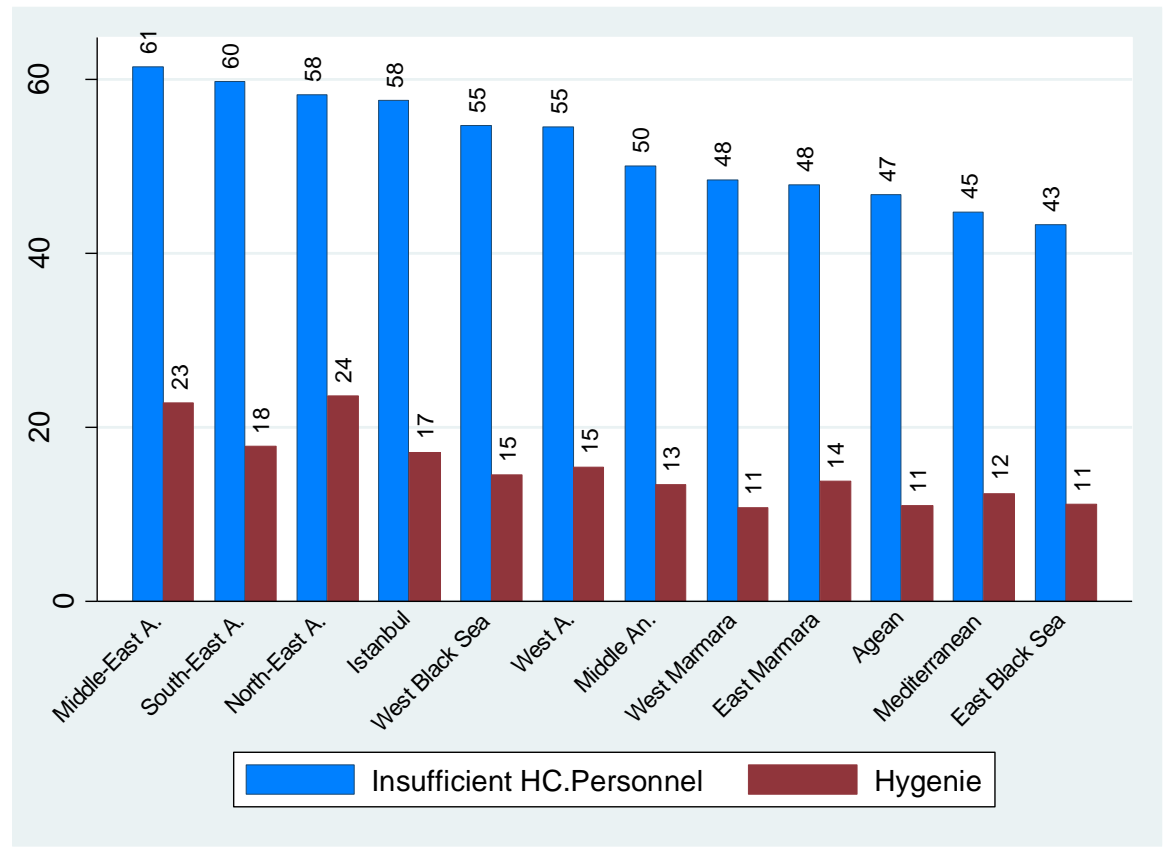

Figure 4: Organization Problems by Region 


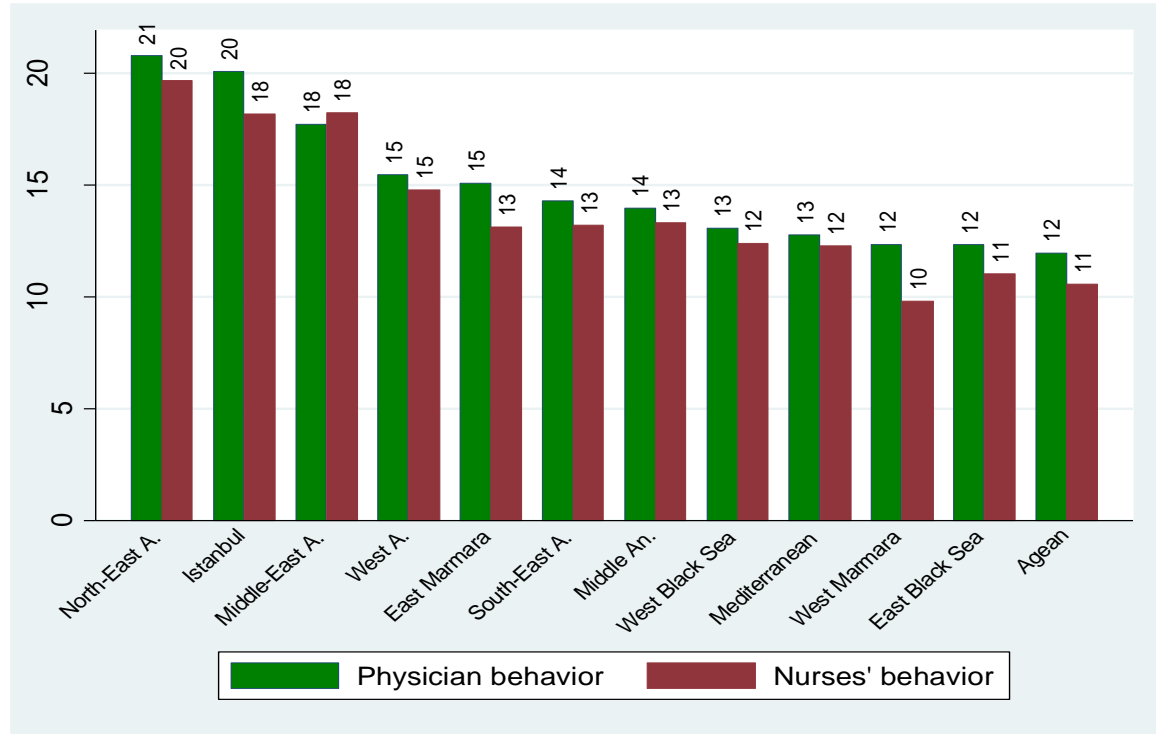

Figure 5: Problems due to Health Care Staff by Region

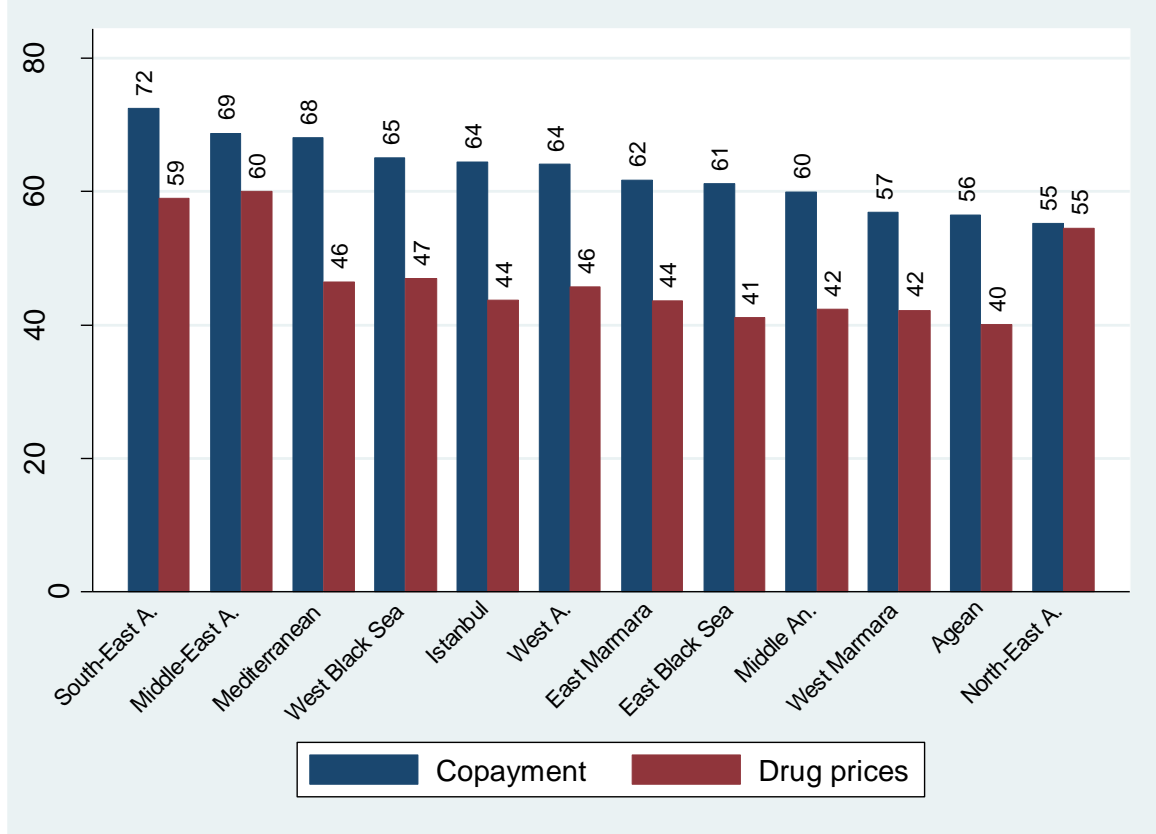

Figure 6: Problems due to Health Care Service Costs by Region

Figure 6 shows the percentage of people that find drug prices high and consider copayments to be not affordable. When we compare the percentage of people who experience troubles in various domains of health care services, the highest percentages are observed among price-related problems. Approximately $63 \%$ of people find co-payments to be problematic, and 
$46 \%$ of people are uncomfortable with drug prices. There do not exist significant discrepancies across regions if we take North-East, South-East, and Middle-East Anatolia out of consideration. As before, South-East, North-East, and Middle East Anatolia are the top 3 regions that are uncomfortable with drug prices.

\section{DISCUSSION AND CONCLUSIONS}

In this study, we examined regional disparities in subjective health assessment, health care utilization, and satisfaction with health services. Before the HTP, health inputs had been distributed unequally across regions in Turkey, which led to inequity in access to health services and health outcomes. One primary tool to narrow inequities across regions is the allocation of health inputs. In this sense, supply-side interventions such as the distribution of physical capital and human capital are of great importance. We show that the most disadvantaged regions, the North-East, South-East and Middle-East Anatolia, benefited the most from the HTP, as the most significant percentage increases in the number of physicians, hospital beds and healthcare institutions are observed in these regions. Our results suggest that the historically poor regions, North-East, South-East, and Middle-East Anatolia, benefited more from the Green Card scheme compared to other regions. In that sense, HTP was successful in reducing regional inequalities concerning access to health insurance and health-care services.

However, our findings also indicate that while accounting for health insurance and supply-side factors in regression analyses reduces the variation across regions to some extent, there still exists substantial heterogeneity among regions in terms of subjective health, utilization and satisfaction with healthcare services. Moreover, the disadvantaged North-East, Middle-East, and South-East Anatolia regions have lower utilization, lower satisfaction with 
healthcare, and lower subjective health scores in 2013 even after accounting for a wide range of control variables.

Although differences in utilization patterns across regions could be interpreted as an indication of inequity in health care access (Waters, 2000), utilization differences may stem from other factors such as differences in cultural acceptability of the health services provided.(Thiede et al., 2007)

Our findings suggest that a significant cause of differences across regions was not related to the quantity of healthcare resources but rather the differences in the quality of services. According to Appendix Figures B.9, B.10, and B.112, North-East, Middle-East and South-East regions fared better than many other regions in terms of quantity of health inputs. However, we observe that North-East, Middle-East and South-East Anatolia regions have greater problems in all aspects of health-care service delivery from appointment to hygiene, from organizational aspects to behaviour of health workforce.

Further, although the Green Card scheme intended to increase access to health care services for the poor, $60 \%$ of respondents reported issues with co-payment and drug prices which was the most common problem across all regions in relation to the cost of healthcare services. Ideally, the combination of different insurance schemes under the roof of Social Security Institution enables equal access to the same benefits. However, in reality, the green card holders need to renew their status and prove their eligibility. This could hinder their utilization of healthcare services from time to time.

Overall, the findings suggest health insurance coverage is not enough to close the gap between less developed and more developed regions. Indeed, studies show that co-payment 
might be hindering the utilization of health-care services in less developed regions even if the health benefits of these services far exceed cost.(Banerjee and Duflo, 2011; Dupas, 2009)

A major finding of this study is that although the underdeveloped regions benefited quantitatively higher from the HTP, there is considerable quality differential across regions. There were more problems reported in less developed regions of Turkey in terms of physicians' and nurses' attitudes towards patients, which can easily erode the much-needed trust between healthcare staff and patients. The variation in quality of services has also been reported in India (Das amd Hammer, 2005) and elsewhere (Arsenault et al., 2019; Chaudhury et al., 2006). Our results are in line with the findings for Tanzania in which following expansion of access to healthcare facilities to rural populations, large variation in the quality of care provided is reported.(Leonard and Masatu, 2007) The variation in the quality of services in certain regions of Turkey can help explain underutilization in these areas.

Further research is necessary for shedding light on what drives regional disparities in Turkey. For example, with The HTP, primary care and preventive care systems are combined into the family practice system. This started to operate with a profit margin which was for free before the HTP.

There are certain limitations of this study. We used a one-year cross-section survey to examine the regional health disparities, which means that we were unable to watch the same regions over time. Unfortunately, in recent versions of life satisfaction surveys, TurkStat does not provide region or province information. Another limitation is that the survey was conducted in 2013 which is almost eight years ago. 
Notwithstanding limitations, our study provides new evidence on the regional differences and disparities in health care system following health system reforms. In order to realize the full benefits of UHC, regional disparities need to be examined and systematically addressed.

Ethical Approval: No need for ethics approval as secondary data is used in this study.

Authors' Contributions: ZU conceived the study, analyzed the data and wrote the first draft. AT redrafted the paper. Both authors reviewed and approved the final version.

Funding and Acknowledgment: No funding is received. Many thanks to Rifat Atun for his comments on earlier versions of this study.

Conflict of Interest Statement: There is no conflict of interest to disclose regarding this study.

\section{REFERENCES}

Arsenault, C., English, M., Gathara, D., Malata, A., Mandala, W., \& Kruk, M. E. (2019). Variation in competent and respectful delivery care in Kenya and Malawi: A retrospective analysis of national facility surveys. Tropical Medicine and International Health, 25,442-453.

Atun, R. (2015). Transforming Turkey's health system-lessons for universal coverage. New England Journal of Medicine, 373,1285-1289.

Atun, R., Aydın, S., Chakraborty, S., Sümer, S., Aran, M., Gürol, I., Nazlığlu, S., Özgülcü, Ş., Aydoğan, Ü., Ayar, B., Dilmen, U., Akdağ, R. (2013). Universal health coverage in Turkey: Enhancement of equity. The Lancet, 382,65-99.

Baicker, K., Taubman, S. L., Allen, H. L., Bernstein, M., Gruber, J. H., Newhouse, J. P., Schneider, M.D., Wright, B.J., Zaslavsky, A.M., Finkelstein, A. N. (2013). The Oregon experiment-effects of medicaid on clinical outcomes. New England Journal of Medicine, 368,1713-1722.

Banerjee, A., Duflo, E. (2011). Poor economics: A radical rethinking of the way to fight global poverty. New York: Public Affairs.

Chaudhury, N., Hammer, J., Kremer, M., Muralidharan, K., Rogers, F. H. (2006). Missing in action: Teacher and health worker absence in developing countries. Journal of Economic Perspectives, 20,91-116.

Das, J., Hammer, J. (2005). Which doctor? Combining vignettes and item response to measure clinical competence. Journal of Development Economics, 78,348-383. 
Dupas, P. (2009). What matters (and what does not) in households' decision to invest in malaria prevention? American Economic Review, 99,224-230.

Engström, S., Foldevi, M., Borgquist, L. (2001). Is general practice effective? A systematic literature review. Scandinavian Journal of Primary Health Care, 19,131-144.

Grogger, J., Arnold, T., León, A. S., Ome, A. (2014). Heterogeneity in the effect of public health insurance on catastrophic out-of-pocket health expenditures: The case of Mexico. Health Policy and Planning, 30,593-599.

Gruber, J., Hendren, N., Townsend, R. M. (2014). The great equalizer: Health care access and infant mortality in Thailand. American Economic Journal: Applied Economics, 6,91-107.

Hacettepe University Institute of Population Studies. (1994). Demographic Health Survey 1993, Ankara.

Hacettepe University Institute of Population Studies. (2004). Demographic Health Survey 2003, Ankara.

Karra, M., Fink, G., Canning, D. (2016). Facility distance and child mortality: A multi-country study of health facility access, service utilization, and child health outcomes. International Journal of Epidemiology, 46,817-826.

King, G., Gakidou, E., Imai, K., Lakin, J., Moore, R. T., Nall, C., Ravishankar, N., Vargas, M., Rojo, M.M.T., Ávila, J.E.H., Ávila, M.H., Llamas, H.H. (2009). Public policy for the poor? A randomised assessment of the Mexican universal health insurance programme. The Lancet, 373,1447-1454.

Knaul, F.M., Arreola-Ornelas, H., Wong, R., Lugo-Palacios, D. G., Méndez-Carniado, O. (2018). The effect of Seguro Popular de Salud on catastrophic and impoverishing expenditures in Mexico, 2004-2012. Salud Pública de México, 60,130-140.

Knaul, F.M., González-Pier, E., Gómez-Dantés, O., García-Junco, D., Arreola-Ornelas, H., Barraza-Lloréns, M., Sandoval, R., Caballero, F., Avila, M.H., Juan, M., Kershenobich, D., Nigenda, G., Ruelas, E., Sepulveda, J., Tapia, R., Soberon, G., Chertorivski, S., Frenk, J. (2012). The quest for universal health coverage: Achieving social protection for all in Mexico. The Lancet, 380, 1259-1279.

Knaul, F.M., Wong, R., Arreola-Ornelas, H., Méndez, O., Bitran, R., Campino, A. C., Rathe, M. (2013). Household catastrophic health expenditures: a comparative analysis of twelve Latin American and Caribbean Countries. Mexican Health Foundation, International Development Center. Boston: Harvard University Press.

Kutzin, J. (2013). Health financing for universal coverage and health system performance: Concepts and implications for policy. Bulletin of the World Health Organization, 91,602-611.

Leonard, K.L., Masatu, M.C. (2007). Variations in the quality of care accessible to rural communities in Tanzania: Some quality disparities might be amenable to policies that do not necessarily relate to funding levels. Health Affairs, 26,380-392.

Masters, S.H., Burstein, R., Amofah, G., Abaogye, P., Kumar, S., Hanlon, M. (2013). Travel time to maternity care and its effect on utilization in rural Ghana: A multilevel analysis. Social Science and Medicine, 93,147-154.

Ministry of Health. (2010). Health Statistics Yearbook 2008, Ankara.

Ministry of Health. (2014). Health Statistics Yearbook 2013, Ankara.

Sahin, I., Ozcan, Y.A., Ozgen, H. (2011). Assessment of hospital efficiency under health transformation program in Turkey. Central European Journal of Operations Research, 19,19-37.

Stokes, J., Gurol Urganci, I., Hone, T., Atun, R. (2015). Effect of health system reforms in Turkey on user satisfaction. Journal of Global Health, 5,020403. 
Tangcharoensathien, V., Patcharanarumol, W., Ir, P., Aljunid, S. M., Mukti, A. G., Akkhavong, K., Mills, A. (2011). Health-financing reforms in southeast Asia: challenges in achieving universal coverage. The Lancet, 377,863-873.

Thiede, M., Akweongo, P., McIntyre, D. (2007). Exploring the dimensions of access. D. McIntyre, G. Mooney (Ed.), The economics of health equity içinde (103-124. ss.). Cambridge: Cambridge University Press.

Tirgil, A., Dickens, W. T., Atun, R. (2019). Effects of expanding a non-contributory health insurance scheme on out-of-pocket healthcare spending by the poor in Turkey. BMJ Global Health, 4,e001540.

Tirgil, A., Gurol Urganci, I., Atun, R. (2018). Early experience of universal health coverage in Turkey on access to health services for the poor: Regression kink design analysis. Journal of gGobal Health, 8,020412.

TurkStat. (2013, October 10). Life Satisfaction Survey. http://tuik.gov.tr/MicroVeri/YMA_2013/index.html

TurkStat. (2013, October 10). Province indicators and health. https://biruni.tuik.gov.tr/ilgosterge/?locale=en

Ugur, Z., Tirgil, A. (2018). Sağl1kta dönüşüm programi ve kamunun sağlık hizmetlerinden memnuniyeti. Ombudsman Akademik, 1,295-327.

Wagstaff, A., Lindelow, M., Jun, G., Ling, X., Juncheng, Q. (2007). Extending health insurance to the rural population: An impact evaluation of China's new cooperative medical scheme. Journal of Health Economics, 28,119.

Waters, H.R. (2000). Measuring equity in access to health care. Social Science and Medicine, 51,599-612.

World Health Organization. (2010). World Health Report, 2010: Health systems financing the path to universal coverage.

Yardim, M.S., Cilingiroglu, N., Yardim, N. (2013). Financial protection in health in Turkey: The effects of the health transformation programme. Health Policy and Planning, 29,177-192. 


\section{Appendix A: Key Reforms under the Health Transformation Program}

- The very first change made with the HTP was to eliminate the involuntary incarceration of patients in hospitals who cannot pay their health care expenditures

- The coverage of the Green Card was expanded to include outpatient, outpatient prescriptions, eye, and dental services.

- A performance-based supplementary payment system was initiated at all MoH facilities.

- Social Insurance Organization hospitals were transferred to $\mathrm{MoH}$, which led to unification of all public health-care provisions in the hand of Ministry of Health.

- Members of both Social Insurance Organization and Bağ-Kur were also given the same status as Government Employees Retirement Fund members in terms of ability to visit university hospitals directly.

- The VAT rate of pharmaceutical products has been reduced from $18 \%$ to $8 \%$, which reduced the burden on patients.

- Different health insurance holders' status with regard to receiving treatment from private health care providers was equalized.

- The extra charges private hospitals made to patients receiving health-care services was standardized and the extra fee which is charged to patients is bounded to be up to $30 \%$ of what private hospitals receive from the Social Security Institution.

- All different health insurance schemes with various benefits packages and financing systems were unified under one umbrella of Social Insurance Organization to reach Universal Health Insurance.

- Those who are younger than 18 years of age included in the coverage of UHI and were eligible to use all health services free of charge

- A contract-based family medicine system across the country was introduced.

- The patients were given a chance to choose their doctors so that health care providers feel more the need to pay more attention to their patients' needs and satisfaction.

- All ambulance services were made available for everyone, including those in rural areas.

- The co-payment system was introduced for outpatient services, doctor visits, and dental care services when obtained at hospitals.

- A full-time practice was made compulsory for university and $\mathrm{MoH}$ personnel. 


\section{Appendix B}

Table B-1. Health Inputs across Regions

\begin{tabular}{|c|c|c|c|c|c|c|}
\hline \multirow[b]{2}{*}{ Region } & \multicolumn{2}{|c|}{ \# Physicians } & \multicolumn{2}{|c|}{ \# of MoH inst. } & \multicolumn{2}{|c|}{ \# of Beds (MoH) } \\
\hline & 2003 & 2013 & 2003 & 2013 & 2003 & 2013 \\
\hline South-East Anatolia & 5888 & 12374 & 65 & 87 & 7395 & 11585 \\
\hline North-East Anatolia & 1988 & 3138 & 36 & 47 & 3100 & 4274 \\
\hline Middle-East Anatolia & 2743 & 4654 & 51 & 55 & 5210 & 6935 \\
\hline West Marmara & 3175 & 5242 & 54 & 51 & 5715 & 5952 \\
\hline Aegean & 15187 & 21343 & 117 & 124 & 16260 & 17683 \\
\hline East Marmara & 5531 & 9088 & 60 & 70 & 7499 & 9914 \\
\hline West Anatolia & 14367 & 19176 & 50 & 51 & 10594 & 11312 \\
\hline Mediterranean & 11994 & 16570 & 91 & 86 & 14021 & 15063 \\
\hline Middle Anatolia & 3033 & 4025 & 71 & 70 & 5648 & 5566 \\
\hline West Black Sea & 5447 & 7998 & 88 & 93 & 11093 & 11038 \\
\hline East Black Sea & 2822 & 4073 & 61 & 65 & 6635 & 6482 \\
\hline Istanbul & 22291 & 26094 & 45 & 55 & 14601 & 15465 \\
\hline
\end{tabular}


Table B-2 Ordered Probit Regression Results

\begin{tabular}{|c|c|c|c|c|c|c|}
\hline & $\begin{array}{c}\text { SWH } \\
\text { (1) }\end{array}$ & $\begin{array}{l}\text { SWH } \\
(2)\end{array}$ & $\begin{array}{c}\text { SWH } \\
(3)\end{array}$ & $\begin{array}{c}\text { SHS } \\
(4)\end{array}$ & $\begin{array}{c}\text { SHS } \\
(5)\end{array}$ & $\begin{array}{c}\text { SHS } \\
(6)\end{array}$ \\
\hline $\begin{array}{l}\text { South-East An. } \\
\text { (ref: Middle An.) }\end{array}$ & $\begin{array}{c}-0.22^{* * * *} \\
(0.01)\end{array}$ & $\begin{array}{c}-0.18^{* * * *} \\
(0.01)\end{array}$ & $\begin{array}{c}-0.16^{* * * *} \\
(0.01)\end{array}$ & $\begin{array}{c}-0.17^{* * * *} \\
(0.01)\end{array}$ & $\begin{array}{c}-0.16^{* * * *} \\
(0.01)\end{array}$ & $\begin{array}{c}-0.10^{* * * *} \\
(0.02)\end{array}$ \\
\hline Middle-East An. & $\begin{array}{c}-0.16^{* * * *} \\
(0.01)\end{array}$ & $\begin{array}{l}-0.11^{* * * *} \\
(0.01)\end{array}$ & $\begin{array}{l}-0.13^{* * *} \\
(0.02)\end{array}$ & $\begin{array}{c}-0.30^{* * * *} \\
(0.02)\end{array}$ & $\begin{array}{c}-0.29^{* * * *} \\
(0.02)\end{array}$ & $\begin{array}{c}-0.33^{* * * *} \\
(0.02)\end{array}$ \\
\hline North-East An. & $\begin{array}{c}-0.08^{* * * *} \\
(0.02)\end{array}$ & $\begin{array}{l}-0.04^{* *} \\
(0.02)\end{array}$ & $\begin{array}{l}-0.04^{* *} \\
(0.02)\end{array}$ & $\begin{array}{c}-0.14^{* * *} \\
(0.02)\end{array}$ & $\begin{array}{c}-0.12^{* * * *} \\
(0.02)\end{array}$ & $\begin{array}{c}-0.14^{* * * *} \\
(0.02)\end{array}$ \\
\hline Mediterranean & $\begin{array}{c}-0.07^{* * * *} \\
(0.01)\end{array}$ & $\begin{array}{c}-0.07^{* * * *} \\
(0.01)\end{array}$ & $\begin{array}{l}-0.03^{*} \\
(0.01)\end{array}$ & $\begin{array}{c}-0.13^{* * * *} \\
(0.01)\end{array}$ & $\begin{array}{c}-0.13^{* * * *} \\
(0.01)\end{array}$ & $\begin{array}{c}-0.06^{* * * *} \\
(0.01)\end{array}$ \\
\hline West Marmara & $\begin{array}{l}0.09^{* * *} \\
(0.01)\end{array}$ & $\begin{array}{l}0.09^{* * * *} \\
(0.01)\end{array}$ & $\begin{array}{l}0.12^{* * *} \\
(0.01)\end{array}$ & $\begin{array}{c}-0.08^{* * *} \\
(0.01)\end{array}$ & $\begin{array}{c}-0.08^{* * *} \\
(0.02)\end{array}$ & $\begin{array}{c}-0.06^{* * *} \\
(0.02)\end{array}$ \\
\hline East Marmara & $\begin{array}{l}0.02^{*} \\
(0.01)\end{array}$ & $\begin{array}{c}0.02 \\
(0.01)\end{array}$ & $\begin{array}{l}0.04^{* * *} \\
(0.01)\end{array}$ & $\begin{array}{c}-0.09^{* * * *} \\
(0.01)\end{array}$ & $\begin{array}{c}-0.09^{* * * *} \\
(0.01)\end{array}$ & $\begin{array}{c}-0.07^{* * * *} \\
(0.01)\end{array}$ \\
\hline Istanbul & $\begin{array}{c}0.01 \\
(0.02)\end{array}$ & $\begin{array}{c}0.01 \\
(0.02)\end{array}$ & $\begin{array}{l}0.09^{* * * *} \\
(0.02)\end{array}$ & $\begin{array}{c}-0.20^{* * * *} \\
(0.02)\end{array}$ & $\begin{array}{c}-0.20^{\text {****** }} \\
(0.02)\end{array}$ & $\begin{array}{l}-0.03^{*} \\
(0.02)\end{array}$ \\
\hline Aegean & $\begin{array}{l}0.10^{* * * *} \\
(0.01)\end{array}$ & $\begin{array}{l}0.10^{* * * *} \\
(0.01)\end{array}$ & $\begin{array}{l}0.15^{* * *} \\
(0.01)\end{array}$ & $\begin{array}{c}-0.03^{* * * *} \\
(0.01)\end{array}$ & $\begin{array}{l}-0.03^{* *} \\
(0.01)\end{array}$ & $\begin{array}{l}0.04^{* * *} \\
(0.01)\end{array}$ \\
\hline West An. & $\begin{array}{c}0.01 \\
(0.01)\end{array}$ & $\begin{array}{c}0.01 \\
(0.01)\end{array}$ & $\begin{array}{l}0.11^{* * * *} \\
(0.02)\end{array}$ & $\begin{array}{c}-0.12^{* * * *} \\
(0.02)\end{array}$ & $\begin{array}{c}-0.12^{* * * *} \\
(0.02)\end{array}$ & $\begin{array}{c}0.02 \\
(0.02)\end{array}$ \\
\hline West Black Sea & $\begin{array}{l}0.08^{* * * *} \\
(0.01)\end{array}$ & $\begin{array}{l}0.08^{* * * *} \\
(0.01)\end{array}$ & $\begin{array}{c}0.08^{* * * *} \\
(0.01)\end{array}$ & $\begin{array}{c}0.02 \\
(0.01)\end{array}$ & $\begin{array}{c}0.02 \\
(0.01)\end{array}$ & $\begin{array}{l}-0.00 \\
(0.02)\end{array}$ \\
\hline East Black Sea & $\begin{array}{l}0.06^{* * *} \\
(0.02)\end{array}$ & $\begin{array}{l}0.06^{* * *} \\
(0.02)\end{array}$ & $\begin{array}{l}0.05^{* * * *} \\
(0.02)\end{array}$ & $\begin{array}{c}0.02 \\
(0.02)\end{array}$ & $\begin{array}{c}0.02 \\
(0.02)\end{array}$ & $\begin{array}{l}-0.03 \\
(0.02)\end{array}$ \\
\hline Health Insurance & - & + & + & - & + & + \\
\hline Healthcare Inputs & - & - & + & - & - & + \\
\hline Baseline controls & + & + & + & + & + & + \\
\hline $\begin{array}{l}\text { Pseudo R-squared } \\
\mathrm{N}\end{array}$ & $\begin{array}{c}0.069 \\
196,203\end{array}$ & $\begin{array}{l}0.070 \\
192,875\end{array}$ & $\begin{array}{l}0.071 \\
192,875\end{array}$ & $\begin{array}{l}0.053 \\
196,203\end{array}$ & $\begin{array}{l}0.054 \\
192,875\end{array}$ & $\begin{array}{l}0.055 \\
192,875\end{array}$ \\
\hline
\end{tabular}

Notes: SWH stands for Satisfaction with Health, SHS stands for Satisfaction with Health Services. Std. errors are clustered at household level and provided in ( ), *** $\mathrm{p}<0.01, * * \mathrm{p}<0.05, * \mathrm{p}<0.10$. The reference category is Middle Anatolia region which includes the Aksaray, Kayresi, Kırıkkale, Kırşehir, Nevşehir, Niğde, Sivas and Yozgat provinces. All models include baseline controls. Baseline Controls: gender, age, age squared, 5 household income bracket dummy variables, employment status dummy variables (categories: employed, unemployed, doing house-care, student, retired, unable to work, other employment status), marital status dummy variables (categories: married, divorced/separated, widowed, single), educational attainment dummy variables (categories: primary school or less, secondary school graduate, high school graduate, university or more), and satisfaction from relationship with friends. Health insurance categories include Social Insurance Organization for formal sector employees, Government Employees Retirement Fund for retired civil servants, Active Civil Servants Insurance Fund for civil servants in work and their dependents, Bağ-Kur for artisans, self-employed and agricultural workers and Green Card Scheme for the poor with the reference category of no insurance. Healthcare inputs include number of physicians per thousand persons, number of public healthcare institutions per thousand persons, number of public hospital beds per thousand persons. 


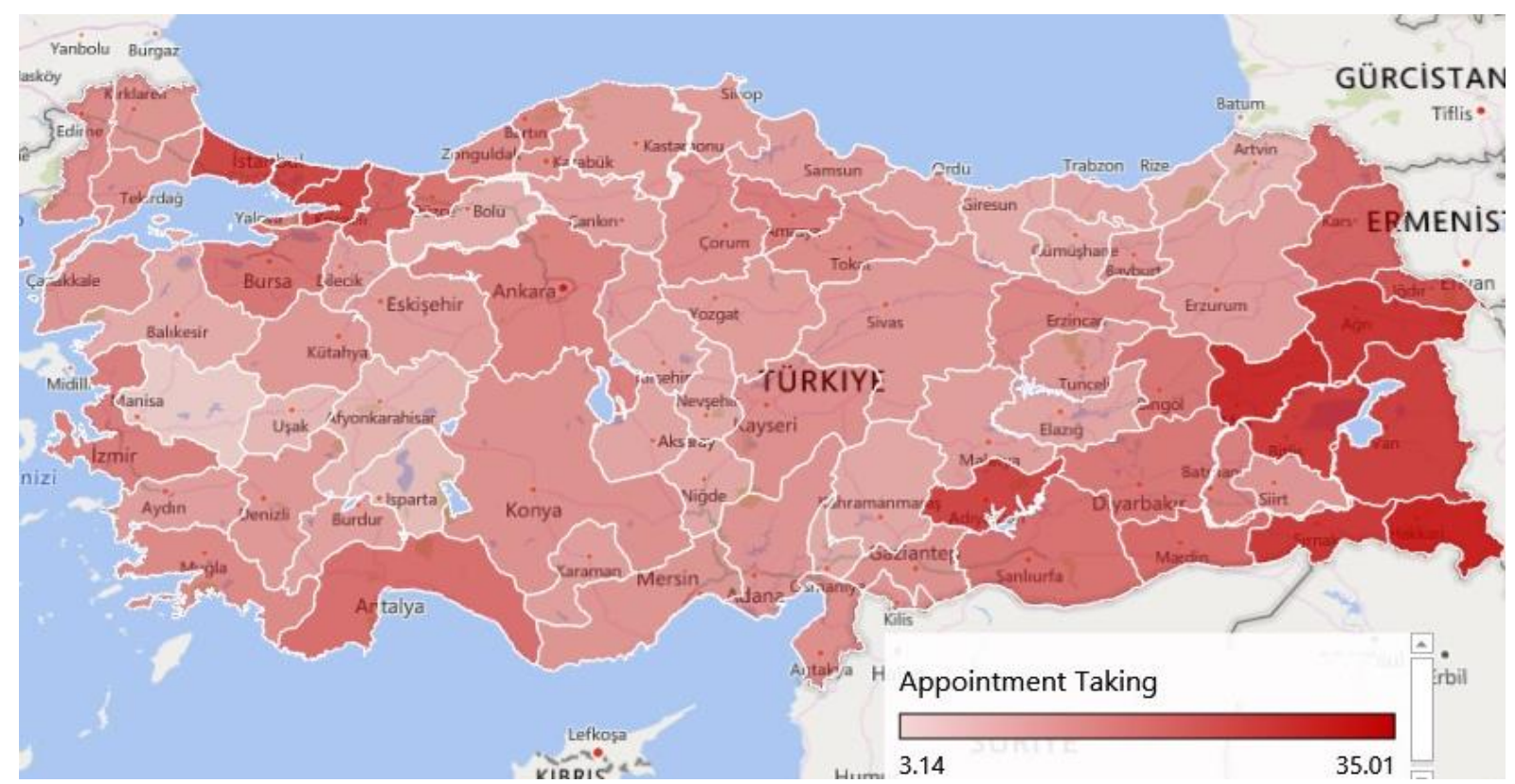

Figure B-1. \% of People Having Problem with Taking Appointment

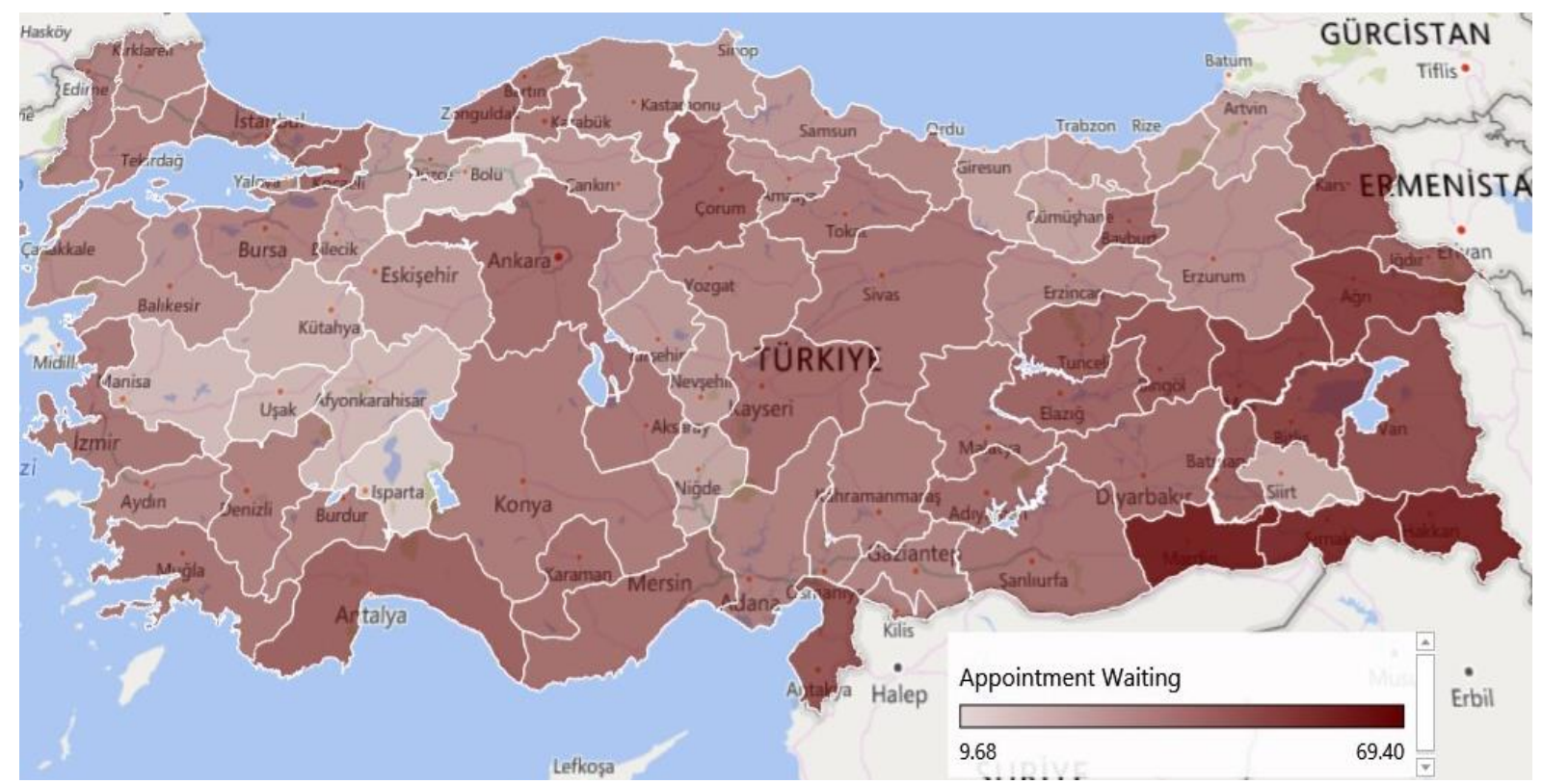

Figure B-2. \% of People Having Problem with Appointment Waiting Time 


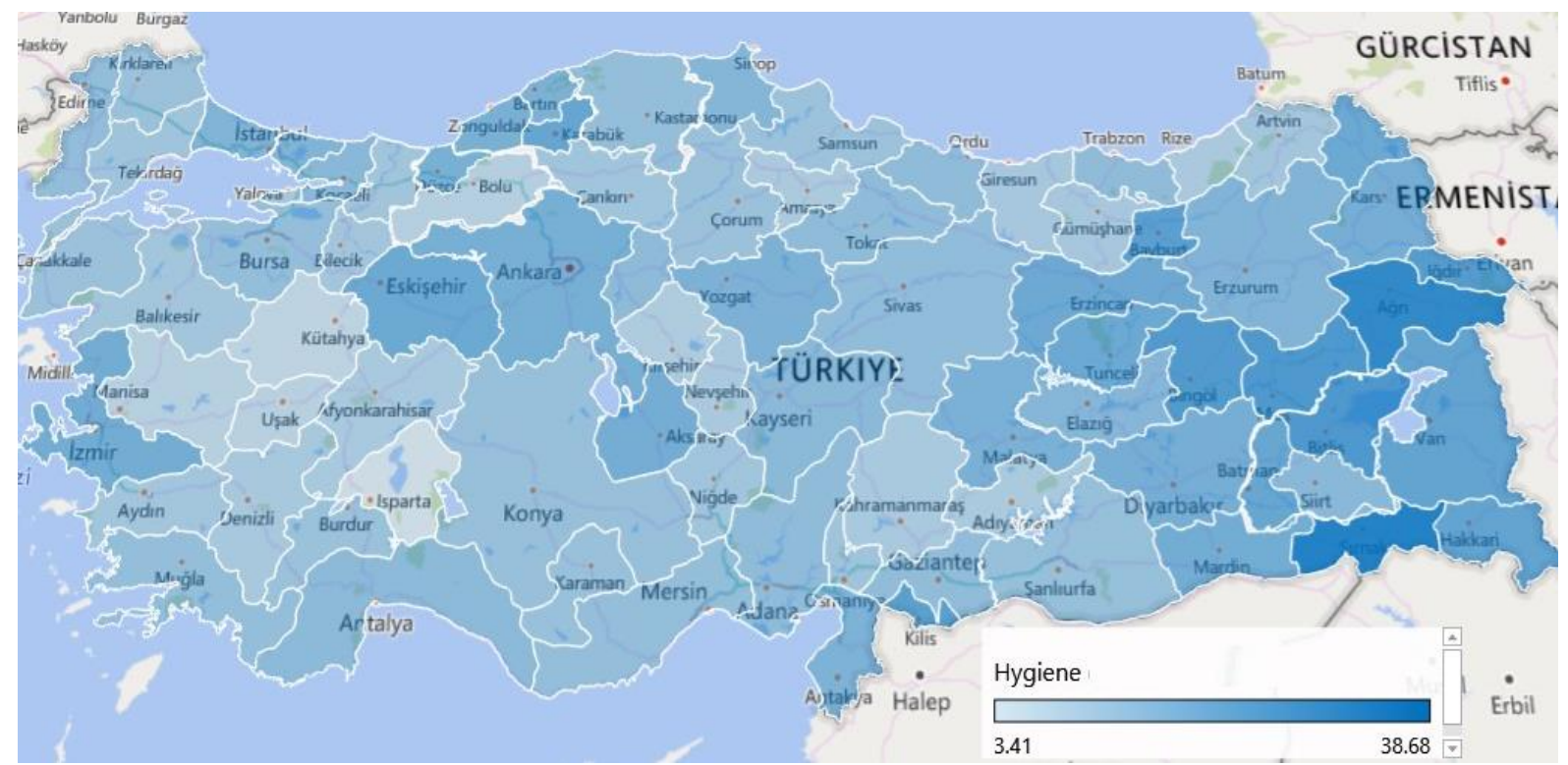

Figure B-3. \% of People Having Problems with Hygiene in Healthcare Institutions

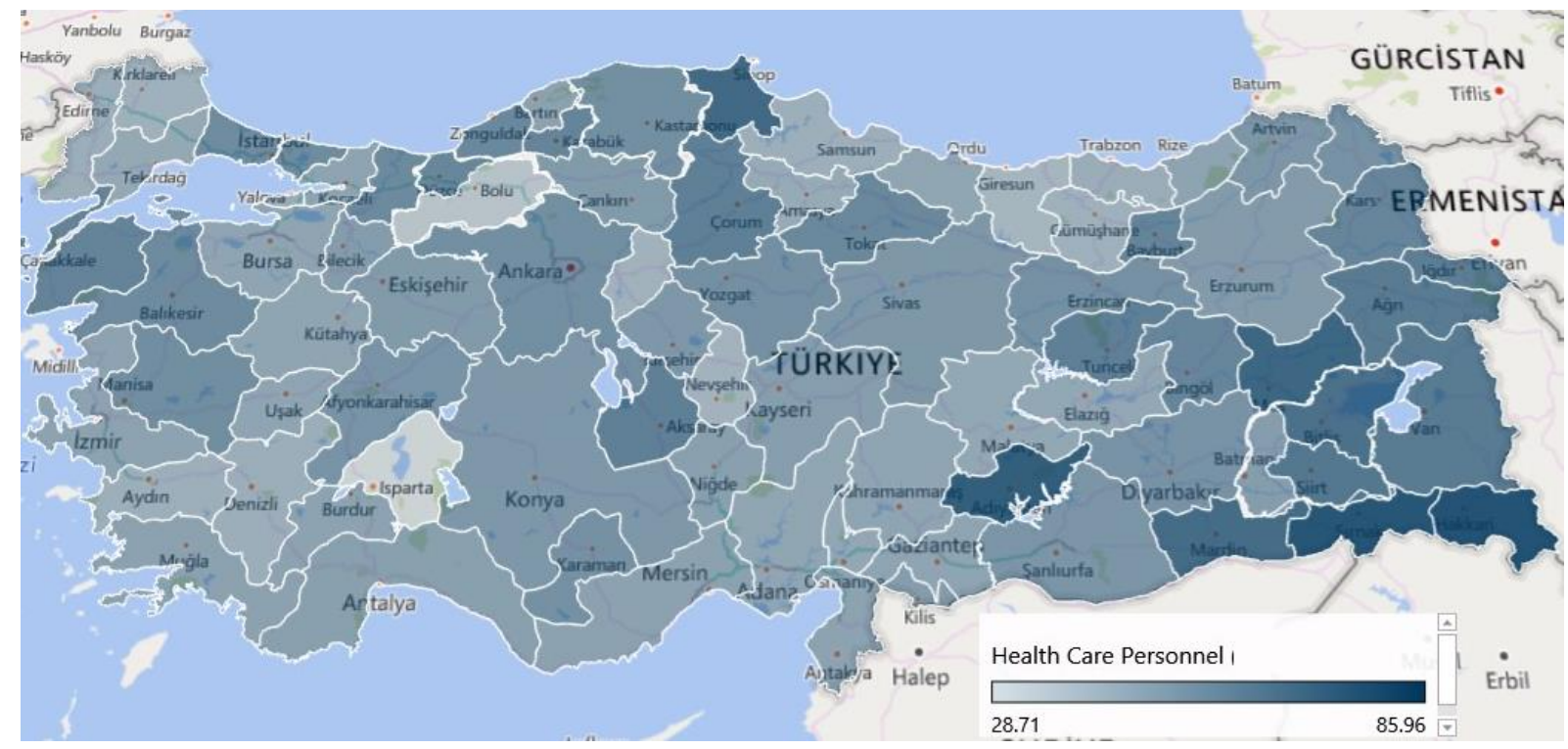

Figure B-4. \% of People Who Finds Healthcare Personnel Insufficient 


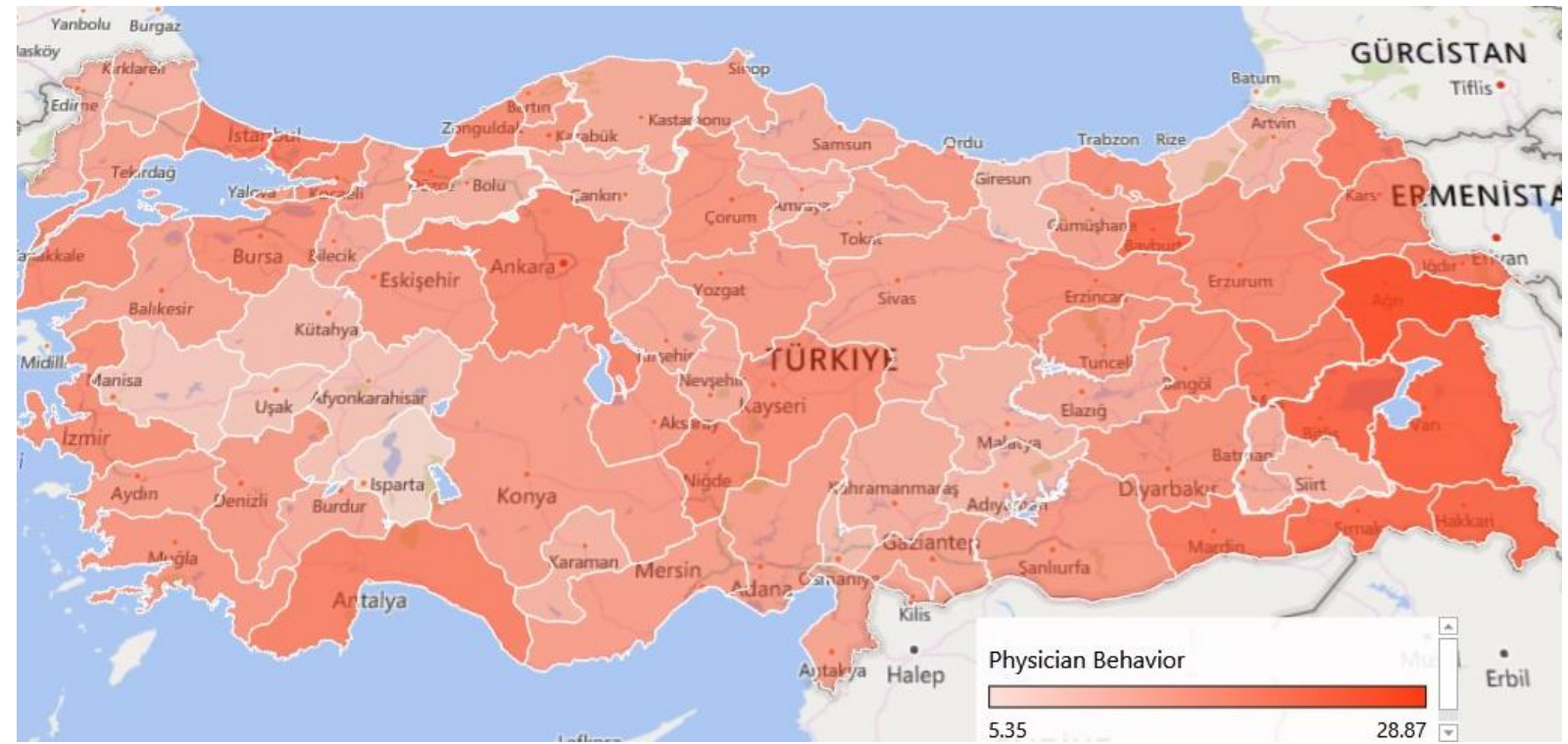

Figure B-5. \% of People Experiencing Problems due to Physician Behaviour

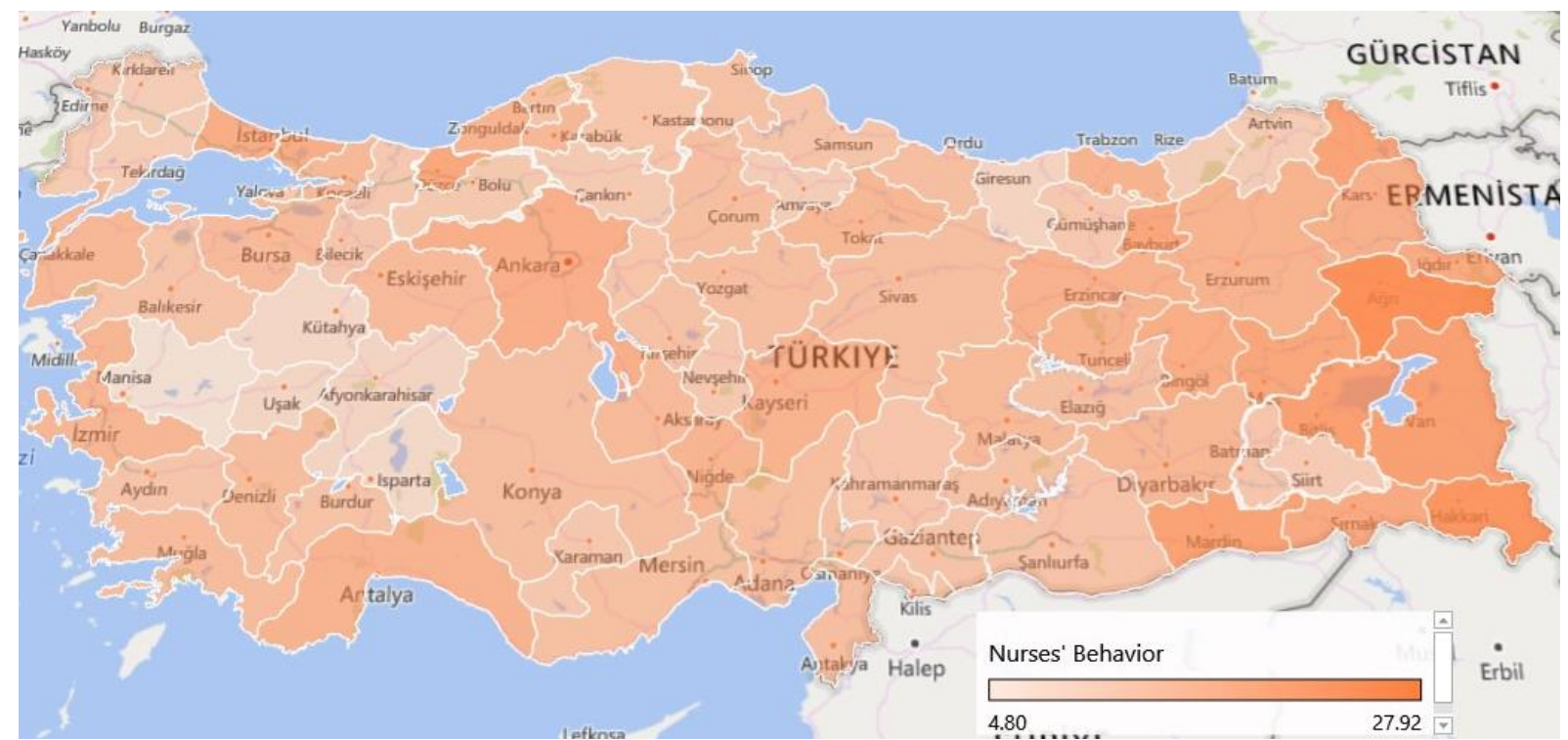

Figure B-6. \% of People Experiencing Problems due to Nurses' Behaviour 


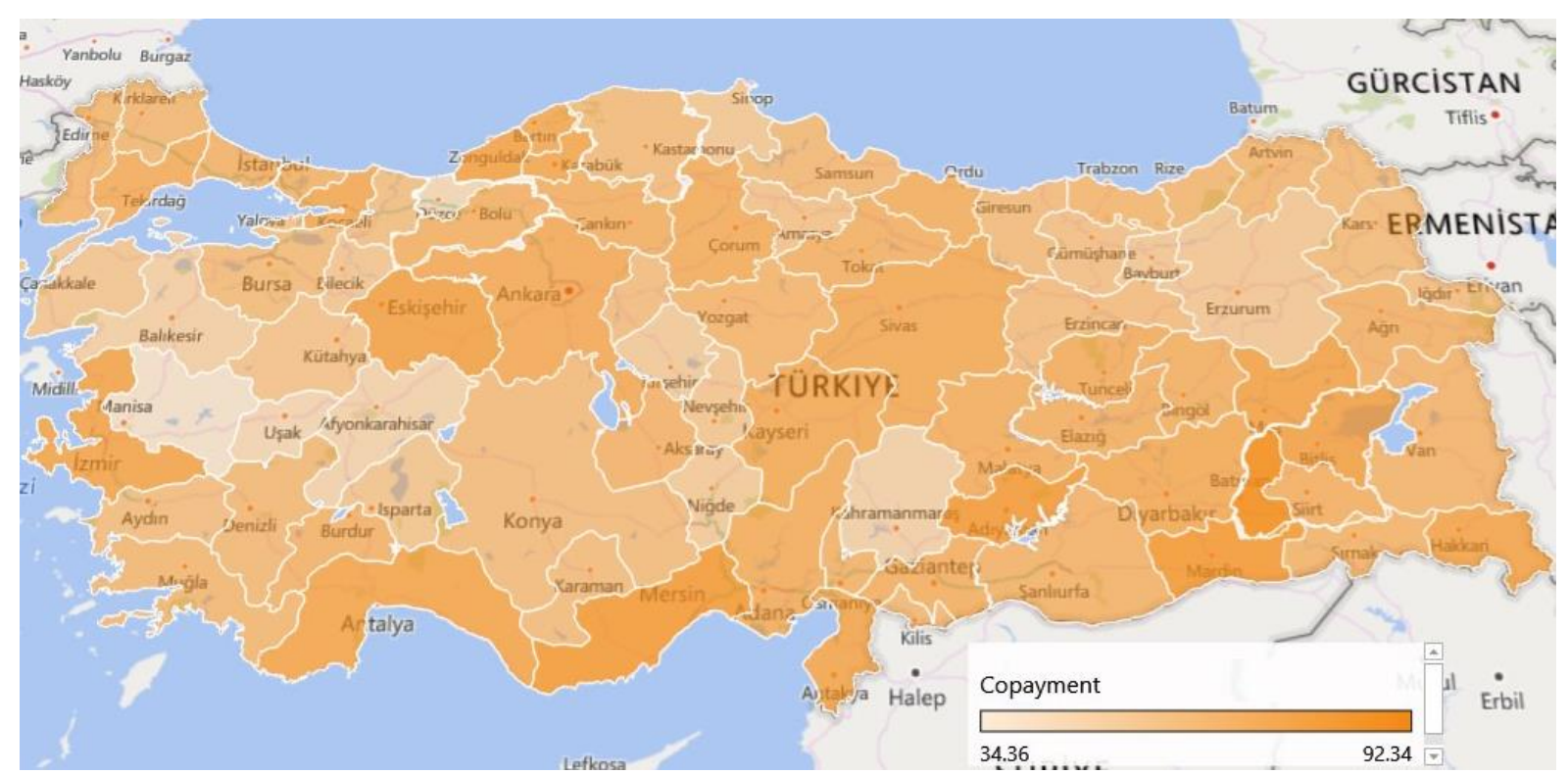

Figure B-7. \% of People Having Issues with Co-payment

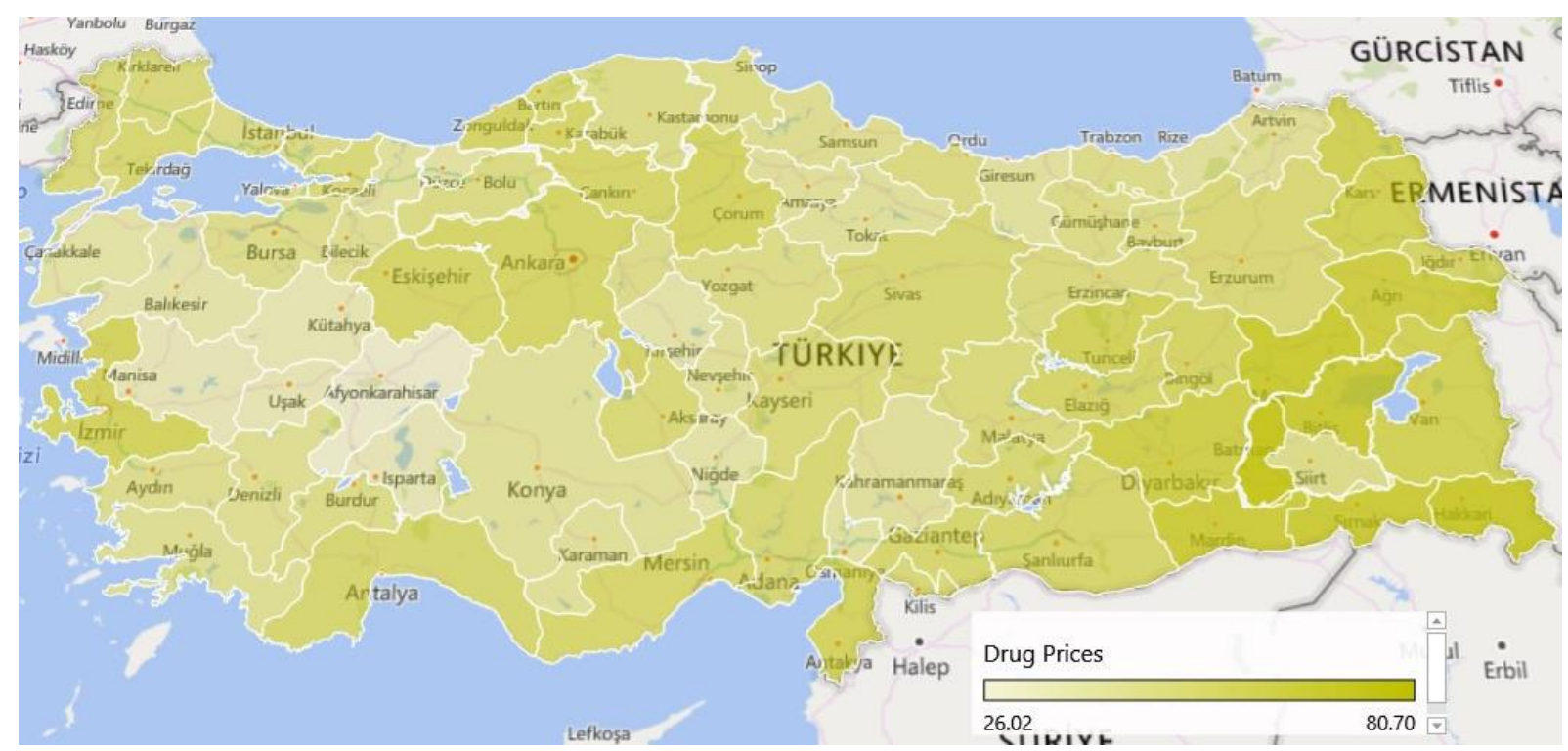

Figure B-8. \% of People Having Issues with Drug Prices 


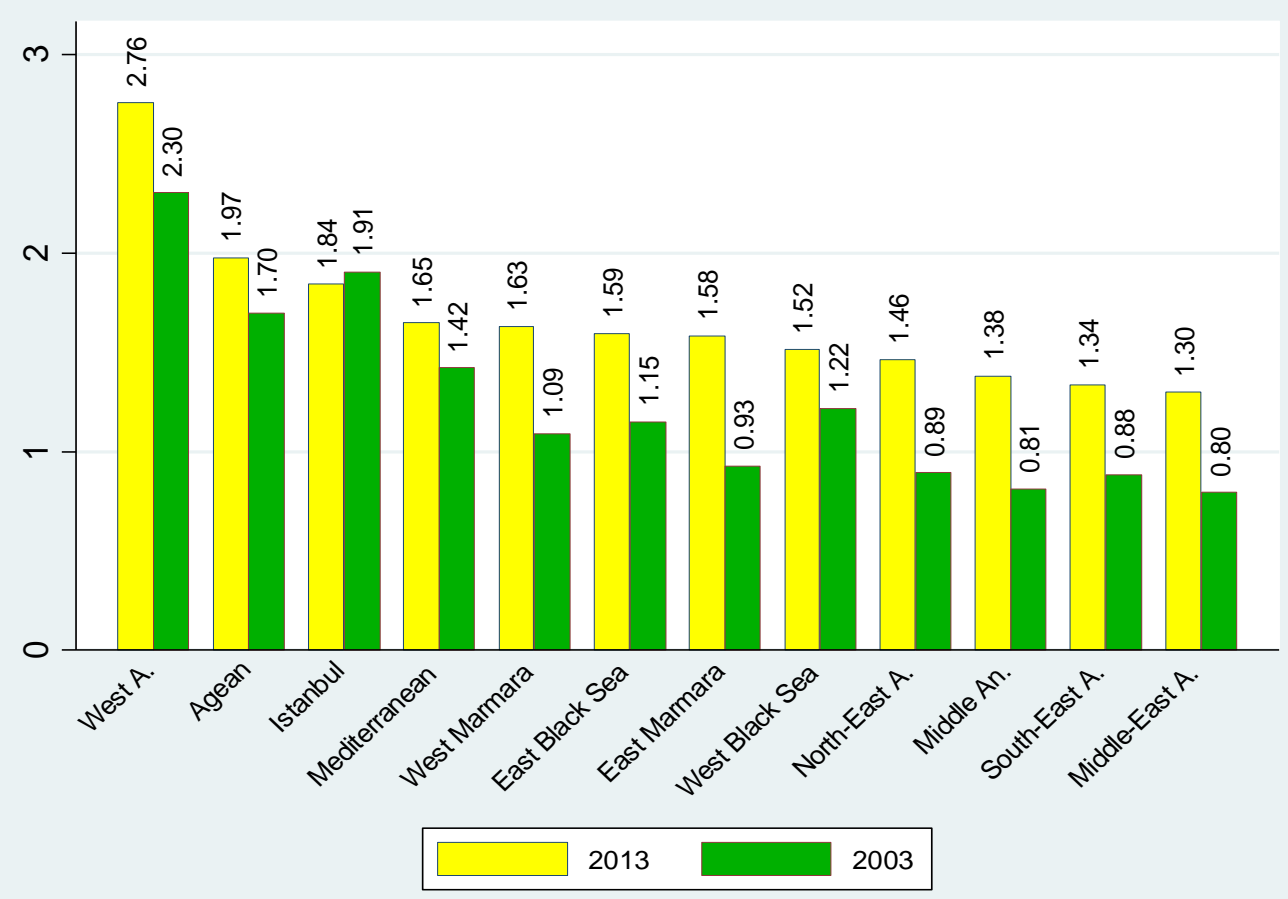

Figure B-9. Number of Physicians ptp across Regions

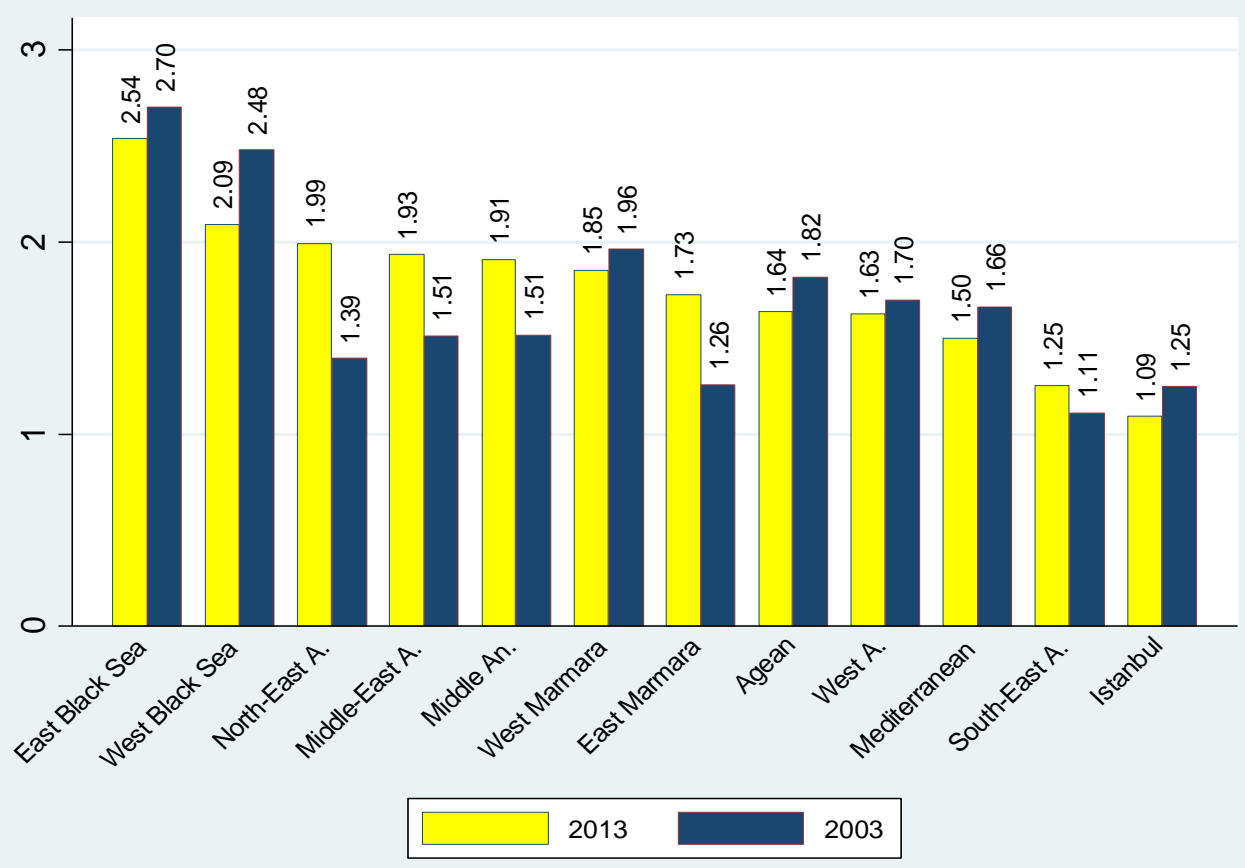

Figure B-10. Number of Hospital Beds ptp across Regions 


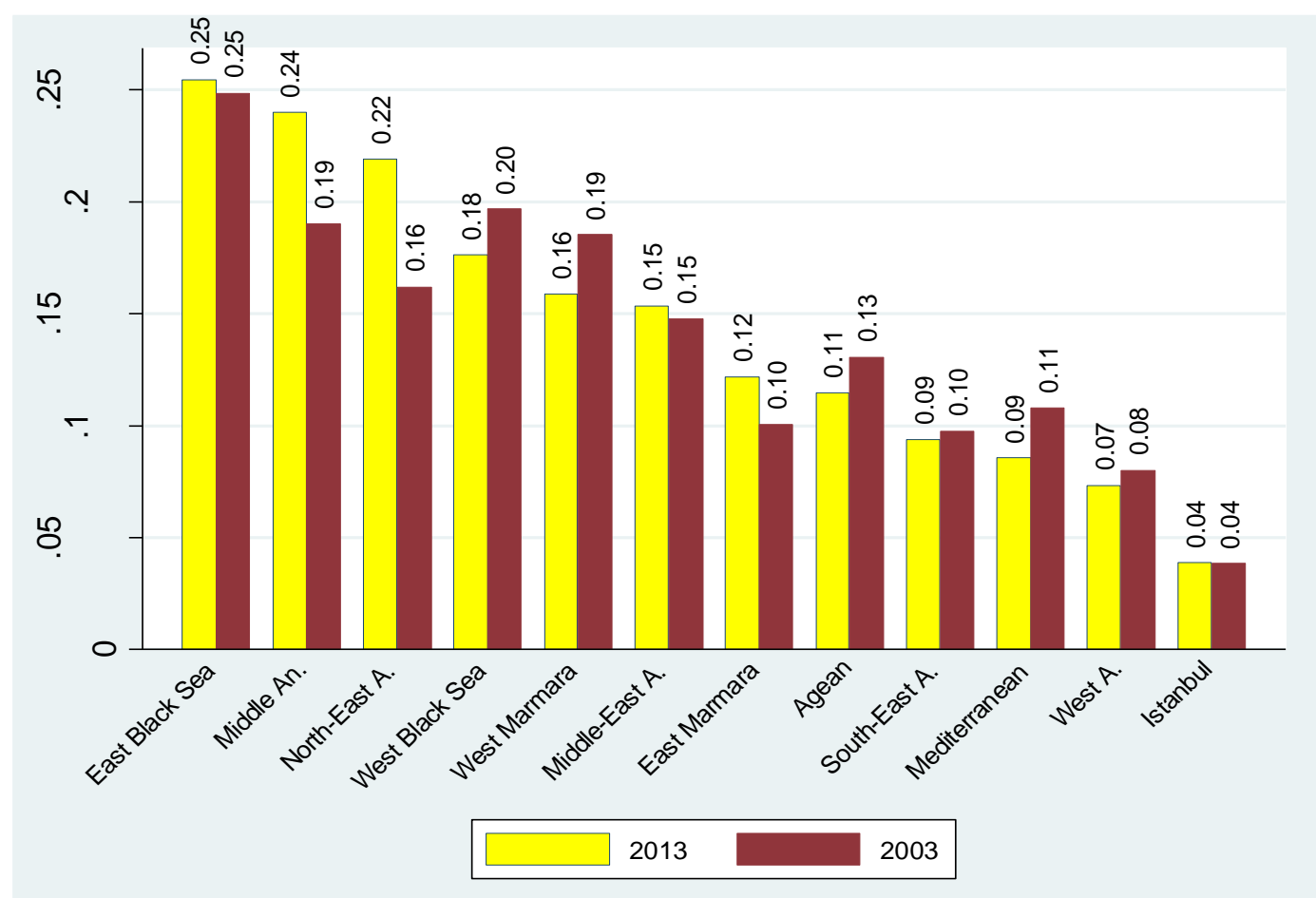

Figure B-11. Number of Health-care Institutions ptp across Regions 